\title{
Emerging functional materials based on chemically designed molecular recognition
}

\author{
Wei Chen ${ }^{2 \dagger}$, Xiaohua Tian ${ }^{1 \dagger}$, Wenbo He ${ }^{1 \dagger}$, Jianwei $\mathrm{Li}^{3^{*}}$, Yonghai Feng ${ }^{1}$ and Guoqing Pan ${ }^{1 *}$ (D)
}

\begin{abstract}
The specific interactions responsible for molecular recognition play a crucial role in the fundamental functions of biological systems. Mimicking these interactions remains one of the overriding challenges for advances in both fundamental research in biochemistry and applications in material science. However, current molecular recognition systems based on host-guest supramolecular chemistry rely on familiar platforms (e.g., cyclodextrins, crown ethers, cucurbiturils, calixarenes, etc.) for orienting functionality. These platforms limit the opportunity for diversification of function, especially considering the vast demands in modern material science. Rational design of novel receptor-like systems for both biological and chemical recognition is important for the development of diverse functional materials. In this review, we focus on recent progress in chemically designed molecular recognition and their applications in material science. After a brief introduction to representative strategies, we describe selected advances in these emerging fields. The developed functional materials with dynamic properties including molecular assembly, enzymelike and bio-recognition abilities are highlighted. We have also selected materials with dynamic properties in contract to traditional supramolecular host-guest systems. Finally, the current limitations and some future trends of these systems are discussed.
\end{abstract}

Keywords: Molecular imprinting, Affinity screening, Dynamic combinatorial chemistry, Dynamic materials, Enzymatic catalysis, Cell recognition

\section{Introduction}

Molecular recognition often is a fundamental event proceeding advanced functions in living systems [1]. Molecular recognition in biochemical processes often involves interactions between host and guest molecules, e.g. receptor-ligand, antibody-antigen, sugar-lectin, DNAprotein, RNA-ribosome [2-4]. In biochemical systems, molecular recognition involves noncovalent interactions that include hydrogen bonds, coordinate bonds,

\footnotetext{
*Correspondence: Jianwei.li@utu.fi; panguoqing@ujs.edu.cn

†Wei Chen, Xiaohua Tian and Wenbo He these authors contributed equally to this work

${ }^{1}$ Institute for Advanced Materials, School of Materials Science

and Engineering, Jiangsu University, Zhenjiang 212013, Jiangsu, China

${ }^{3}$ Hainan Provincial Key Lab of Fine Chem, Key Laboratory of Advanced

Materials of Tropical Island Resources of Ministry of Education, Hainan

University, Haikou 570228, China

Full list of author information is available at the end of the article
}

hydrophobic forces, $\pi-\pi$ interactions, van der Waals forces and electrostatic effects. The complementarity of these interactions provides molecular specificity, which is crucial for specific biological responses like cell signalling, intracellular cascades, cell behaviours and all subsequent biological functions.

An attractive application of molecular specificity is the integration of biological molecular recognition systems (e.g., natural receptor-ligand or antibody-antigen) in artificial materials for the development of functional materials. However, molecular recognition in the natural receptors used in these devices (antibodies, DNA) has inherent drawbacks [5, 6]. First, their chemical and physical stability and shelf life are limited, which seriously restrict their applications in non-physiological environments. Second, the isolation and purification from nature or biochemical synthesis are often expensive, time-consuming and

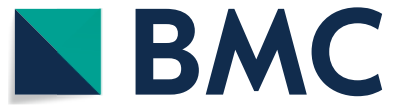

(c) The Author(s) 2020. This article is licensed under a Creative Commons Attribution 4.0 International License, which permits use, sharing, adaptation, distribution and reproduction in any medium or format, as long as you give appropriate credit to the original author(s) and the source, provide a link to the Creative Commons licence, and indicate if changes were made. The images or other third party material in this article are included in the article's Creative Commons licence, unless indicated otherwise in a credit line to the material. If material is not included in the article's Creative Commons licence and your intended use is not permitted by statutory regulation or exceeds the permitted use, you will need to obtain permission directly from the copyright holder. To view a copy of this licence, visit http://creativeco mmons.org/licenses/by/4.0/. The Creative Commons Public Domain Dedication waiver (http://creativecommons.org/publicdomain/ zero/1.0/) applies to the data made available in this article, unless otherwise stated in a credit line to the data. 
labour-intensive. Finally, the conditions required for many practical applications in field of material science are more demanding compared to the those in nature. These disadvantages necessitate scientists to seek more durable and robust receptor-like substitutions [7].

By mimicking the mechanism of nature receptors, chemical combination of noncovalent interactions with spatially and functionally complementarity would help to create synthetic receptor mimics that are comparable to natural molecular recognition $[6,8]$. Supramolecular host-guest interactions represent one of the earliest non-natural molecular recognition [9-11]. By the use of macrocycle hosts (i.e., cyclodextrins, crown ethers, cucurbiturils, calixarenes.) and their corresponding guests [12-16], dynamic polymeric gels are obtained with functions that include responsiveness, self-healing and elastomeric properties [17]. These systems have spearheaded the development of a new generation of materials. However, these traditional host-guest chemistry are relatively limited in number and function, and relatively few have found advanced applications [18]. With increasing demand for multiple functionality in the development of advanced funtional materials, researchers in material science have turned their attention to chemically designed molecular recognition systems [12, 19-21].

In this review, we will introduce several typical chemical strategies for the synthesis of novel receptor-like mimics which imitate the molecular recognition systems in nature and their applications as functional materials. Natural molecular recognition systems, such as DNA/ RNA, antigen-antibody, sugar-lectin, etc., will not be included. We will first elaborate the chemical methods for exploring new synthetic receptors including molecular imprinting, affinity screening, and dynamic combinatorial chemistry (DCC) strategies. Then, the latest progress of these molecular recognition mechanisms in material design as well as their emerging applications will be recapitulatively reviewed and discussed. In the end, we will give a perspective of this field, in particular, the future development of materials based on novel molecular recognition systems. Due to the limited space, we could not completely cover all literatures, but the latest representative examples will be discussed. We hope this paper will not only provide the researchers a panoramic view of chemically designed molecular recognition, but also inspire them to develop new materials for advanced applications in various fields including energy, environment, biomedicine and so on.

\section{Chemically designed molecular recognition Molecular imprinting strategy}

Molecular imprinting (MI) is a chemical technique to create template-shaped cavities in polymer matrices with memory of the template molecules to be used for molecular recognition $[8,22-28]$. In contrast to the other approaches to molecular recognition discussed in this review, molecular imprinting depends on the use of both physical and chemical interactions between the target molecules and functional monomers, to generate a molecularly complementary material or material interface [24]. The typical molecular imprinting process involves the self-assembly of template molecules and functional monomers via either non-covalent interactions or reversible covalent bonds, the resulting complexes subsequently being copolymerized with a suitable cross-linker. After removal of the templates from the obtained cross-linked polymer network, molecular recognition sites complementary in shape, size and functionality to the template molecules are formed (Fig. 1) [7]. To date, molecular imprinting has proven to be the most efficient and versatile technique for incorporating specific molecular recognition sites into polymers leading to polymeric artificial receptors, i.e., the molecularly imprinted polymers (MIPs) [29, 30]. In principle, by means of rational molecular design and chemical synthesis, molecular imprinting allows the rapid and inexpensive generation of synthetic receptors towards almost any target molecules.

Since the first MI-based synthetic receptors reported by Wulff et al. in 1972, [31] MIPs with reversible molecular recognition towards various targeted ligands ranging from small organic molecules to macromolecules like peptides and proteins have been readily prepared, mainly benefiting from the molecularly tunable nature of a imprinting process and the flexibility of polymer matrices [32-36]. Further, owing to their high physical stability,

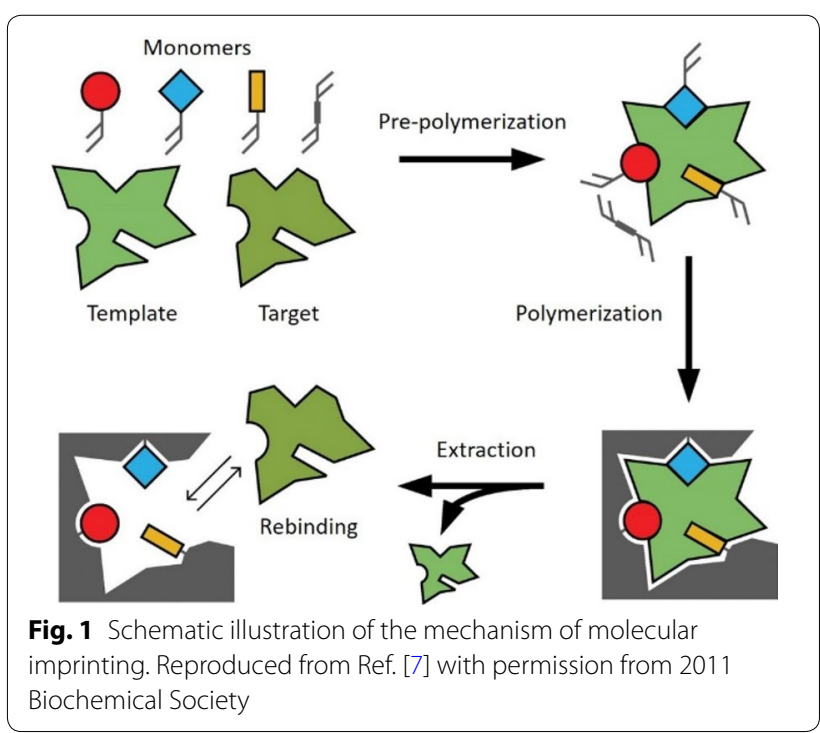


specificity in recognition, and simplicity in synthetic process, molecular imprinting has found use in a wide range of applications encompassing the fields of separation processes (i.e., chromatography, capillary electrophoresis, solid phase extraction, and membrane separation), immunoassays, antibody mimics, artificial enzymes, sensors, catalysis, organic synthesis, drug delivery, drug development, and even bio-imaging [33, 37-39]. As is known, molecular recognition in MIPs occurs by a "lock and key" mechanism that is similar to natural receptorligand interactions. Thus, it is worth highlighting the increasing success of $\mathrm{MI}$ in biomolecular recognition and bio-related applications. The recently reported research in this regard will be discussed in detail in the following sections.

\section{Affinity screening strategy}

Recent studies on interactions between biomolecules and synthetic polymers reveal that polymeric nanoparticles or linear copolymers with special functions and components could exhibit high binding property and even selectivity to biomolecules like peptides, proteins and carbohydrates [40-47]. Schrader and co-work-ers first attempted to synthesize a library of random statistical linear copolymers for the discovery of protein affinity [40]. Then, Shea and co-workers expanded this concept to cross-linked polymeric nanoparticles [42]. The mechanism is based on optimizing the selection and proportion of various functional monomers that are predisposed towards favourable interactions with the target molecules. After screening from a library of synthetic polymer nanoparticles or linear copolymer incorporating a diverse pool of functional monomers, receptor-like candidates with high affinity and selectivity to targeted biomacromolecules can be readily obtained (Fig. 2). Different from molecular imprinting that requires a target template for receptor synthesis, such kind of affinity screening strategy mainly relies on adjusting the chemical composition and thus is very promising for massively exploiting synthetic receptors for bio-recognition, in view of the diversity of biomolecules, and in particular, the scarcity of most of the important biomolecules in biological system.

Commonly, the linear copolymers with uniform length and chemical groups could be synthesized by radical polymerization; while these polymer nanoparticles with uniform size and chemical component are obtained through precipitation or emulsion polymerizations in water solution and are purified by dialyzing the resulting colloidal suspension against a large excess of water [48]. The chemical compositions of these copolymer systems are comprised of carbon backbones with randomly distributed functional side chains by radical polymerization of different functional monomers. To obtain random

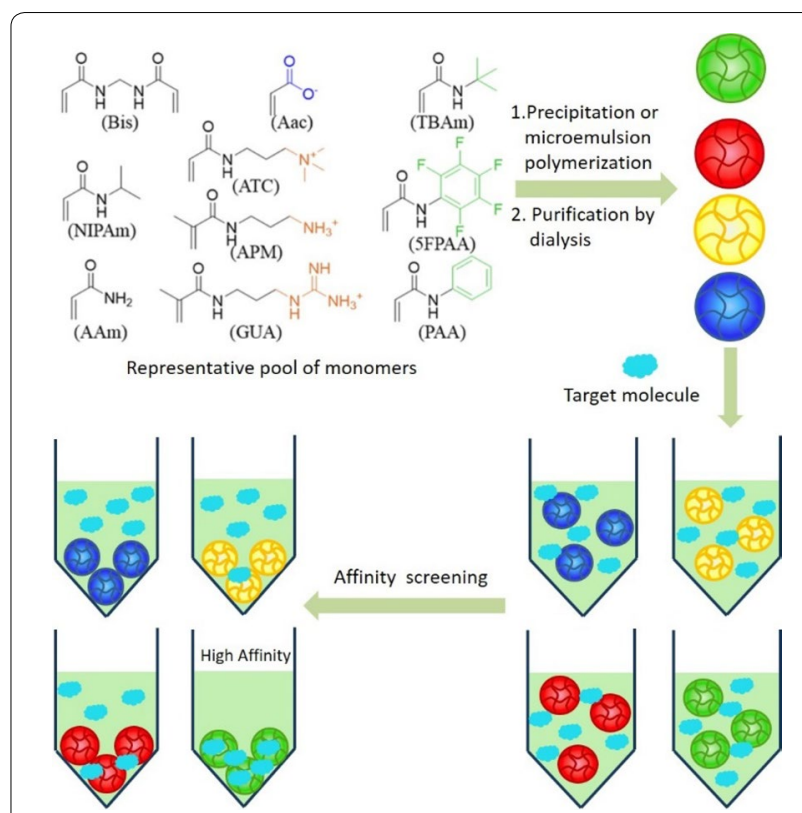

Fig. 2 The mechanism of screening affinity strategy for exploring synthetic polymer nanoparticles with selective affinity. Reproduced from Ref. [44] with permission from 2016 American Chemical Society

stoichiometric incorporation corresponding to the feed ratio, the functional monomers should be either acrylamide or methylacrylamide due to the similar reactivity ratios [49]. Several analytical techniques for evaluating the synthetic receptor-biomolecule affinity, including centrifugation filtration coupled with UV-Vis spectroscopy, quartz crystal microbalance, isothermal titration calorimetry, surface plasmon resonance spectroscopy, peptide activity assays and an ELISA mimic, are necessary for the affinity screening strategy. Perceptibly, the optimized polymer compositions are those that are complementarity in the charge and hydrophobicity of the targeted biomolecule. Although development of this strategy for the exploitation of bio-affinity reagents is in its early stages, current successes has implied the great promise for the development and applications of such low-cost and robust alternatives to the natural antibodies and receptors.

\section{Dynamic combinatorial chemistry (DCC)}

The above strategies have grown into mature branches of exploring innovative receptors for various target molecules. However, they are always followed by a step-wise process of evaluating the synthetic receptors, practically which is time-consuming and sometimes even frustrating. Accordingly, researchers have developed a library-based strategy, DCC, to speed up the discovery processes for receptors [50-54]. In a dynamic combinatorial library $(\mathrm{DCL})$, building blocks react with each 
other in a combinatorial way by linking together through reversible covalent bonds (e.g., disulfides, imines, hydrazones, boronates, etc.), forming a dynamic molecular network $[55,56]$. The concentration distribution of all library members in such complex systems is typically governed by the thermodynamic equilibrium. Because of the reversible nature of DCLs, they are adaptive: for example, introducing a target molecule in a library of receptors may ideally shift the equilibrium to the fittest binding receptors at the expense of the other compounds in the system (Fig. 3) [57]. The DCC approach is endowed with the advantages of both combinatorial chemistry and dynamic covalent chemistry, merging the synthetic and screening process into a single step $[6,58]$. On one hand, the combinatorial reaction forms a pool of library species, providing many possible receptors; on the other hand, the template directed reversible reaction is helpful to sort the effective receptors out from the pool when equilibrium researches at a system level, accelerating the process of exploring receptors.

\section{Functional materials based on chemically designed molecular recognition}

With the rapid development in material engineering and nanoscience, molecular recognition is now playing an increasingly significant role in the design of advanced functional materials. Recently,

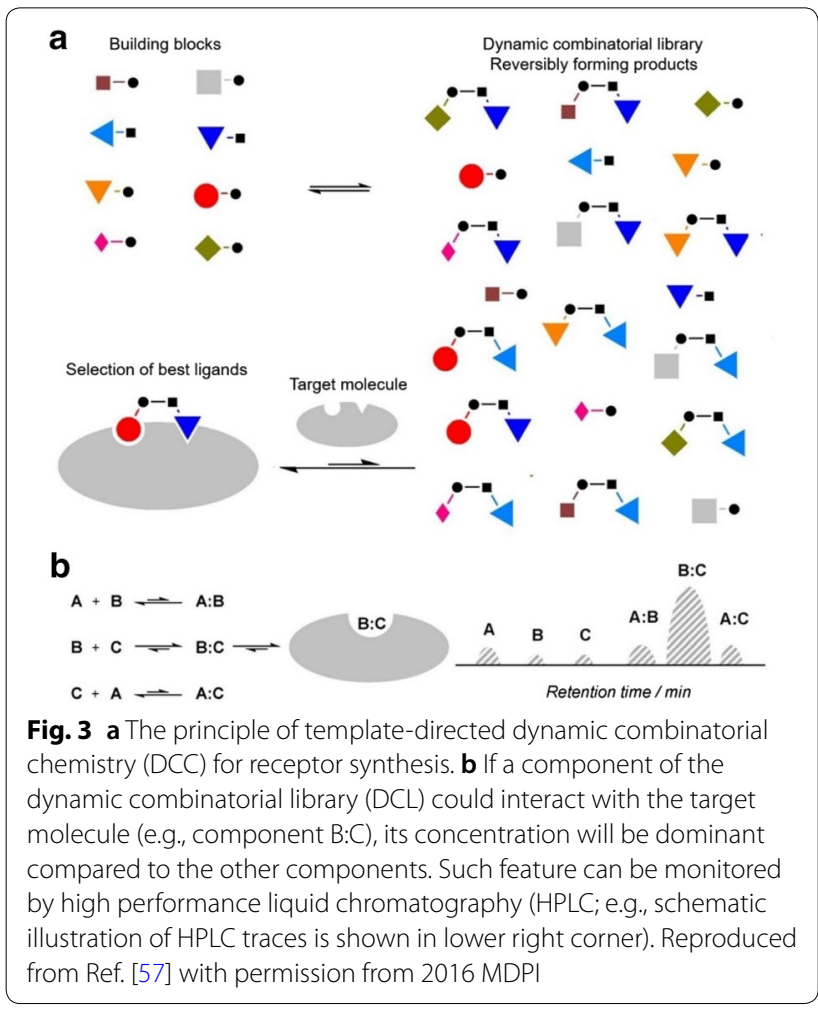

molecular-recognition-based chemical materials with the sizes ranging from nano- to macro-scale and the formats from bulk to interface have been constantly emerging [20, 59-61]. Amongst, functional materials with dynamic properties [62-67], enzyme-like activities [68, 69] and bio-recognition abilities [70-73] have attracted a great deal of attention from researchers in various areas including energy, environment, biomedicine and so on. In this section, we will not give very distinct classification in each subtitle but focus on the above-mentioned three points to describe the most important advances in chemically designed molecular recognition. Given that this review lays emphasis on the chemical design of receptors in the molecular-recognition-based materials, here we just selected and discussed several representative works that are derived from traditional supramolecular hostguest chemistry in the section "Dynamic Materials".

\section{Dynamic materials}

\section{Traditional host-guest chemistry}

For the past few years, traditional supramolecular chemistry based on the molecular recognition of macrocycles (i.e., cyclodextrins, crown ethers, cucurbiturils, calixarenes, etc.) has been an emerging field in material science $[10,17]$. The formation of these supramolecular polymeric networks are attributed to the specific hostguest interactions of the repeating units. The involved building blocks selectively recognize each other, which can define the size, direction and dimension of the resultant polymers that display advanced functions such as stimuli-responsiveness [74], self-healing [75] and rubberlike elastomeric properties [76, 77].

Macroscopic dynamic gels based on the molecular recognition in host-guest supramolecular chemistry have been extensively studied in the past decades. Even so, novel properties based on these traditional molecular systems can be tactfully equipped on materials by sophisticated chemical design. Harada et al. [78] has reported acrylamide-based gels functionalized with either cyclodextrin host rings or hydrocarbon guest. The supramolecular dynamic materials were able to self-assemble in the bulk state. When the size and shape of the host and guest units were changed, different gels can be selectively assembled and be sorted into a distinct macroscopic structure. Taking the above strategy one step further, the same group recently employed two different host-guest inclusion complexes ( $\beta$-cyclodextrin $(\beta C D)$ with adamantane (Ad) or ferrocene $(\mathrm{Fc})$ ) to assemble the polymers together to form a dynamic hydrogel ( $\beta C D$-Ad-Fc) (Fig. 4) [79]. The $\beta C D$-Ad-Fc gel could exhibit self-healing ability when damaged and responded to redox stimuli by expansion or contraction. Moreover, the $\beta C D-A d-F c$ gel showed a redox-responsive shape-morphing effect. 


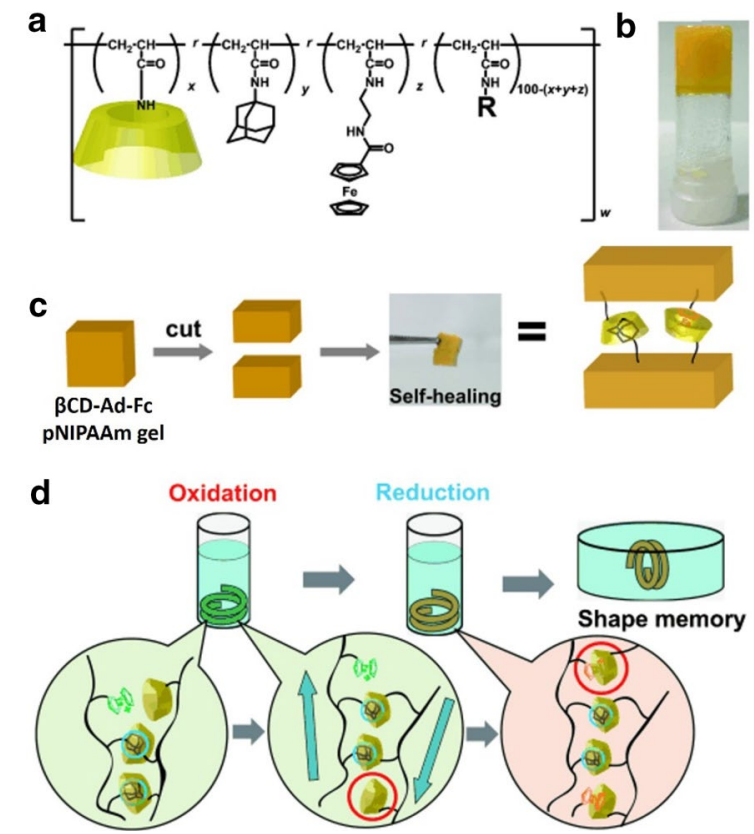

Fig. 4 a The chemical structure of the $\beta C D$-Ad-Fc gels; $x, y$, and $z$ indicate the amount (mol\%) of different units. $\mathbf{b}$ Photograph of the $\beta C D$-Ad-Fc gel. $\mathbf{c}$ Self-healing of the $\beta C D$-Ad-Fcp gel. $\mathbf{a} 4 \times 4 \times 4 \mathrm{~mm}^{3}$ cubic piece of $\beta C D$-Ad-Fc gel was cut in half with a razor edge, and the two cut edges were held together again. Within $2 \mathrm{~h}$ under wet conditions, the gel pieces adhered to form one gel. $\mathbf{d}$ Schematic illustration of the shape-memory mechanism. Reproduced from Ref. [79] with permission from 2015 John Wiley and Sons
This implied that rational design of a supramolecular polymer structure would endow the resultant polymer gel with multifunction. For example, Zhang et al. designed a polymer network containing single-walled carbon nanotubes (SWCNTs) via CD-based molecular recognition. The resultant materials showed bulk electrical conductivity, proximity sensitivity, humidity sensitivity and are able to rapidly self-heal without external stimulus under ambient conditions [77].

In addition to macroscopic gel, recent concerns about host-guest supramolecular dynamic materials also expand to the field of nanoscience. Recently, Huang and his labmates designed a polymeric nano-vesicle by the assembly of a thermo-responsive [3] pseudorotaxane cooperatively complexed by a water-soluble pillar [10] arene and a poly $(N$-isopropylacrylamide) containing paraquat in water [80]. The thermos-responsiveness in the resultant nano-vesicle enable it to be further used for drug delivery under different temperatures. Not limited to nano-vesicles, Zhao's group also achieved to precisely control the biomimetic evolution of these supramolecular assemblies in diverse dimensionalities and geometries. They designed a class of block copolymers containing bioinspired host units with highly-selective capture of

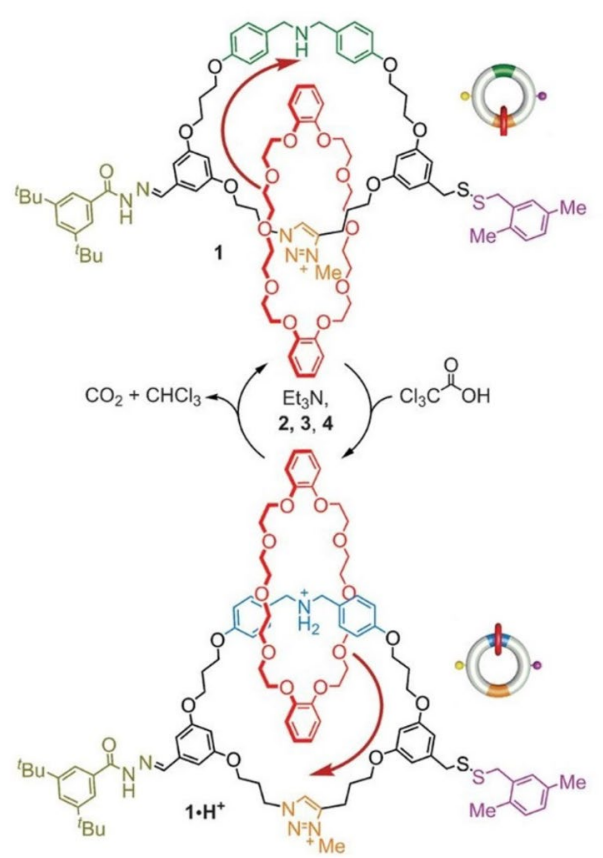

Fig. 5 Operation of rotary motor $1 / 1[\mathrm{H}+]$ using pulses of a chemical fuel. Reagents and conditions: [2] Catenane $1(1.2 \mu \mathrm{mol}, 2 \mathrm{mM})$, $\mathrm{Et}_{3} \mathrm{~N}(23 \mu \mathrm{mol})$, thiol $2(12 \mu \mathrm{mol})$, disulfide $3(60 \mu \mathrm{mol})$, hydrazide $4(4.2 \mu \mathrm{mol}), 2$-methyl-2-butene $(0.94 \mu \mathrm{mol}), \mathrm{CD}_{3} \mathrm{CN}$. Each pulse of fuel contained $96 \mu \mathrm{mol}$ of trichloroacetic acid. Reproduced from Ref. [84] with permission from 2017 the American Association for the Advancement of Science

adenosine-5' ${ }^{\prime}$-triphosphate (ATP) for the assembly [81]. Given this, it offered the chance to achieve ATP-responsive cascading deformations from primary assemblies to sophisticated structures, mimicking biological membrane remodelling. It is really worth to mention that, by means of the molecular recognition in cucurbiturils-based hostguest systems, Zhang et al. put forward a supramolecular modulation method to efficiently control the assembling morphology, including nanorods, octahedron-like nanostructure, helical nanowires, and rectangular nanosheets [82].

Molecular motor is representative of another type of dynamic materials that are responsive for most forms of movement in living organisms [83]. Very recently, Leigh and co-works have powered both rotary and linear synthetic molecular motors based on acid-base oscillations (Fig. 5) [84]. By changing the $\mathrm{pH}$ in the system, the binding affinity of macrocyclic crown ether derivatives on different binding sites on circular or linear tracks was switched. Additionally, the off-on of barriers on the tracks could also be controlled by dynamic covalent chemistry at the different $\mathrm{pH}$. The combination of these factors helped them achieve directional rotation of the 
components in [2, 3] catenane rotary motors. Although this energy ratchet mechanism is simple, it may provide a generally applicable approach to power task performance in molecular nanotechnology.

Obviously, molecular recognition events in the traditional supramolecular chemistry are very efficient for the preparation of dynamic materials. In addition, chemical incorporation of other groups would endow the resultant materials with new functions and structures. However, as is mentioned above, currently host-guest chemistry is relatively rare. The limitations in number and function seriously hindered their development in the design of diverse advance materials that are highly-demand in modern applied material science. From this point of view, molecular recognition materials based on synthetic receptors are more flexible and promising in exploiting new generation of materials for advanced applications.

\section{DCC supramolecular assembly}

The most striking feature of dynamic combinatorial chemistry (DCC) is the dynamic behaviour in molecular assembly. Li et al. recently demonstrated that, by the driven force of molecular recognition, template molecules could trigger the occurrence of self-assembly in a dynamic combinatorial library (DCL), whose result actually is a self-synthesizing nanomaterial. Sequentially, they used $\mathrm{Mg}^{2+}$ as a template to coordinate with carboxylic acid groups of the dimer of the azobenzene-based species in the DCLs and form a self-healing hydrogel that is responsive to an exceptionally large number of stimuli [85]. It may be switched between gel and solution state by light, sequestration or addition of $\mathrm{Mg}^{2+}$, reduction or oxidation, $\mathrm{pH}$ or temperature changes and mechanical energy. These results demonstrate the power of an "ingredients" approach for the development of new selfsynthesizing materials with properties that may be specifically targeted. This example demonstrated that it is not necessary to design the self-assembling molecules. If the right building blocks are provided, the self-assembly process will instruct the system to selectively synthesize the appropriate molecules from these building blocks.

The function of the nanomaterials self-assembled by peptides is highly related to the sequence of the amino acids in the peptide chain. However, it is challenging to design and select suitable self-assembling sequences because of the vast combinatorial space available. Very recently, Prof. Ulijn form the City University of New York has reported a methodology of developing searchable dynamic peptide libraries based on the sequence exchange of unprotected peptides under various conditions (Fig. 6) [86]. The dynamic combinatorial peptide libraries were firstly prepared from building blocks of unprotected homo- and heterodipeptides. The dynamic

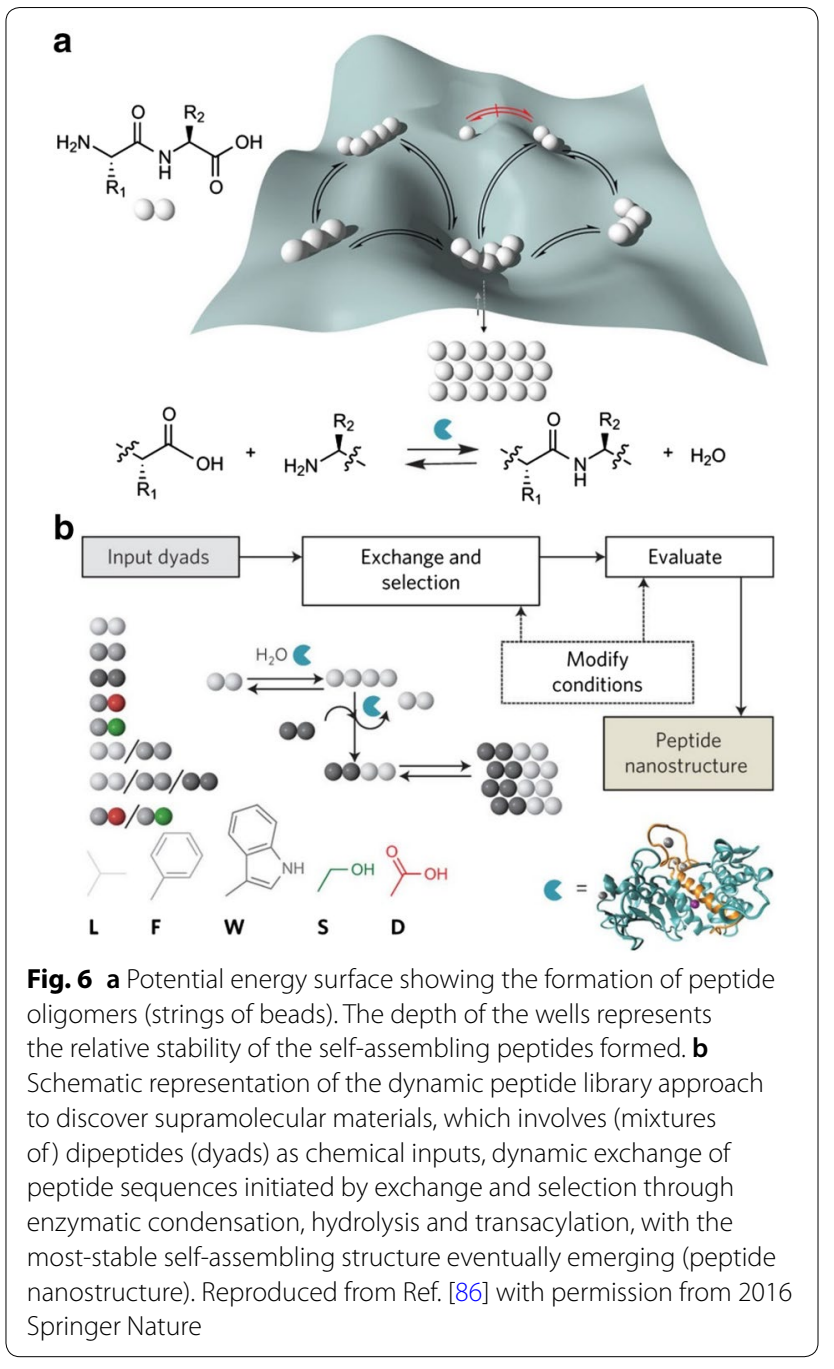

intermolecular conversion and diversification of library species are benefited from continuous enzymatic condensation and hydrolysis. Since the concentration distribution of library species was governed by thermodynamics as the same as the non-enzyme-mediated DCLs, the assembly process was helpful for the amplification of self-assembling candidates. Under various environmental conditions during the selection process, different sequence and consequent nanoscale morphologies are selected. Such automated process opens many possibilities of discovering materials with specific functions from a molecular evolution approach.

Dynamic polymers (i.e., dynamers) are a type of polymers in which monomers are polymerized by either noncovalent or reversible covalent bonds. Prof. Lehn, Prof. Hirsch and Prof. Buhler have cooperated to make biodynamers with tunable properties based on the polycondensation of various categories of amino acid hydrazides with a dialdehyde [87]. In this dynamic system, two types 
of reversible $\mathrm{C}=\mathrm{N}$ bonds (imine and acylhrazone) are both present to produce the polymers. The structures, rates of polymerization and dynamic character have been characterized and they are highly affected by the side chains of the amino acid hydrazides. These results have offered great potential for exploring dynamic materials in both biomedical and bioengineering fields.

\section{Molecular imprinting and affinity screening strategy}

Different from the above molecular recognition systems, molecularly imprinted polymers (MIPs) and affinity-screened synthetic nanoparticles are commonly the receptor aggregates cross-linked by irreversible covalent interactions. Even so, they can also be designed with reversible properties in both nano- and macro-scale. Dynamic materials based on molecular imprinting or affinity screening-based synthetic receptors mainly rely on the combination of stimuli-responsive molecules during the molecular imprinting or synthesis process. Rational choice of functional monomers would endow the resultant MIPs or synthetic nanoparticles with the ability to respond to external stimuli while modulating their affinity for the target molecules and providing a switchable capacity of the binding or releasing processes [88-90]. To date, a variety of intelligent MIPs have been prepared which respond to specific stimuli such as changes in temperature, $\mathrm{pH}$, light, ionic strength, or even the presence of a specific molecule [91, 92]. Amongst, the physiologically related stimuli, in particular, temperature and biomolecules, attracted more attentions due to the potential in bioanalysis, biosensing and drug delivery.

Poly( $N$-isopropylacrylamide) (PNIPAm) has been the most employed synthetic polymer to prepare thermoresponsive MIPs, probably due to its lower critical solution temperature (LCST) is close to the temperature of the human body [93]. By using the thermo-responsive monomer NIPAAm during imprinting, dynamic MIPs with responsive affinity and various formats can be readily prepared. According to this, Pan et al. have prepared a nano-scale protein-imprinted hydrogel in water system by using both negatively and a positively charged functional monomer [94] The lysozyme-imprinted nanogels exhibited size-changing properties under different temperature, thus showing dramatic temperature-dependent rebinding and release characteristics with clear onoff transition around $33{ }^{\circ} \mathrm{C}$. Similarly, Li et al. reported thermo-responsive epitope-imprinted nano-MIPs for specific capture and release of target protein (Fig. 7) [95]. By imprinting the epitope sequence of human serum albumin (HSA), AASQAALGL, the thermo-responsive nano-MIPs could controllably capture the whole target protein HSA from the human plasma at $45^{\circ} \mathrm{C}$ and release it at $4{ }^{\circ} \mathrm{C}$. Such epitope imprinting strategy demonstrated

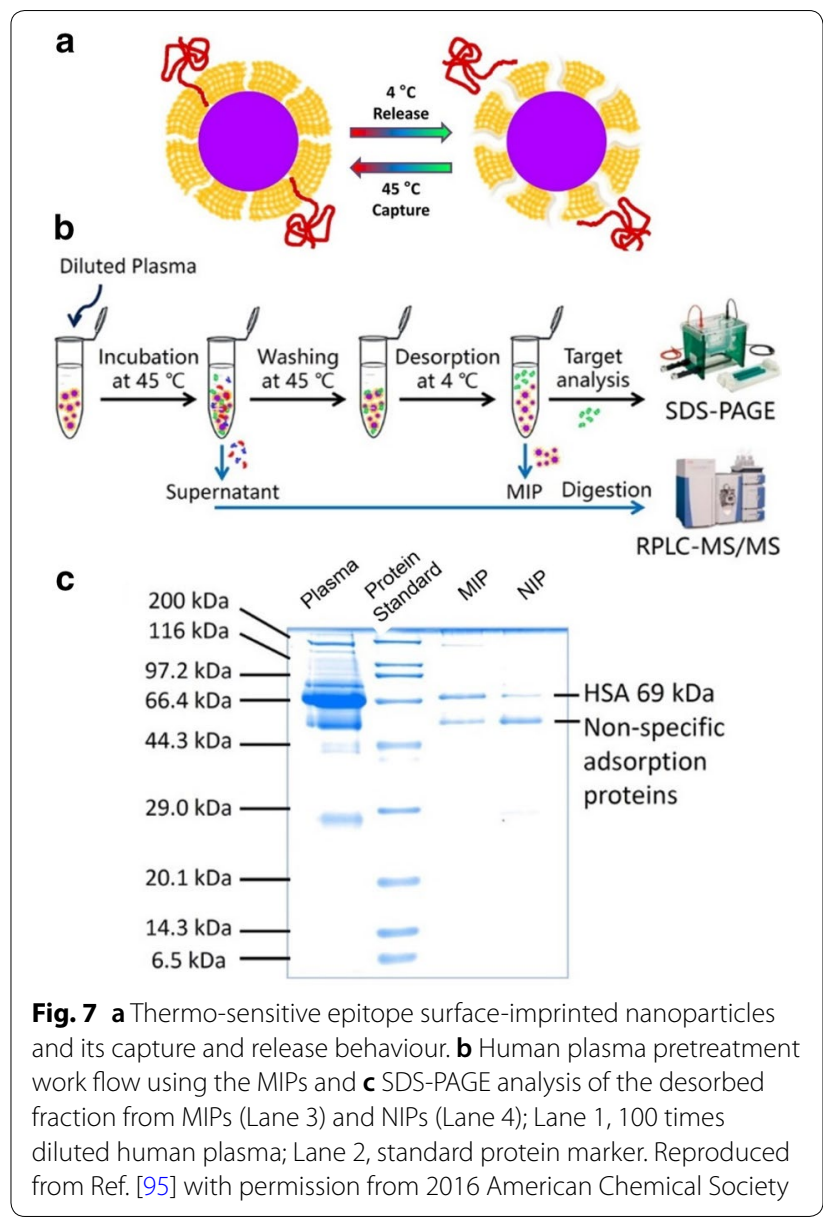

the application potentials of such materials for the recognition of biomolecules that are too expensive to be the templates during imprinting process. Furthermore, by the combined use of thermo- and photo-responsive functional monomers, Zhang's group developed a hydrophilic hollow MIP microsphere with photo- and thermodual-responsive template binding and release behaviours in aqueous media [96]. Wei et al. also reported a selfcleaned electrochemical protein imprinting biosensor basing on a thermo-responsive memory hydrogel [89]. Due to the dynamic surface property response to temperature, the biosensor exhibited a novel self-cleaned ability for bovine serum albumin (BSA) in aqueous media. Not limited to nano-MIPs, recently Pan et al. also synthesized a PNIPAm-based MIP bulk hydrogel layer with thermoresponsive affinity toward cell adhesive peptide RGDS [97]. Due to the thermo-responsive volume-changing and subsequently reversible molecular recognition ability, the RGD peptide imprinted substrate to could be used as cell culture substrate enabling a fast cell sheets harvest. These samples implied that, by using thermoresponsive monomer and molecular imprinting, various 
thermo-responsive synthetic receptors with different formats can be prepared for different applications.

Without special responsive functional monomers, rational chemical design during imprinting process could also realize a target molecule-responsive polymeric network [98]. Takashi et al. first reported a dynamic glycoprotein recognition gel prepared by biomolecular imprinting using lectin and antibody molecules as ligands for tumor-specific marker glycoproteins [99]. The gel could shrink in response to the target glycoprotein, thus enabling the accurate detection tumor-specific markers and showing great potentials as smart devices in sensing systems and for molecular diagnostics. Recently, Bai et al. reported a superaptamer assembly strategy and provided the first example of using protein-specific aptamers to create volume-changing hydrogels with amplified response to the target protein (Fig. 8). The resultant new type of aptamer-based MIP hydrogel could also shrink and the shrinking volume is visible to the naked eye in response to femtomolar concentrations of target protein [100]. Similar to Takashi's mechanism, the extraordinary macromolecular amplification in this work was also attributed to the complex interplay in protein-aptamer supramolecular cross-links and the consequential reduction of excluded volume in the hydrogel. The specific molecular recognition could even be maintained in biological matrices such as urine and tears. In addition, the hydrogels can be dried for long-term storage and be regenerated for use without loss of activity. Obviously, the simple strategy for biomarker detection described in this work offers a promising alternative to traditional analytical techniques that require sophisticated instrumentation and highly trained personnel.

a
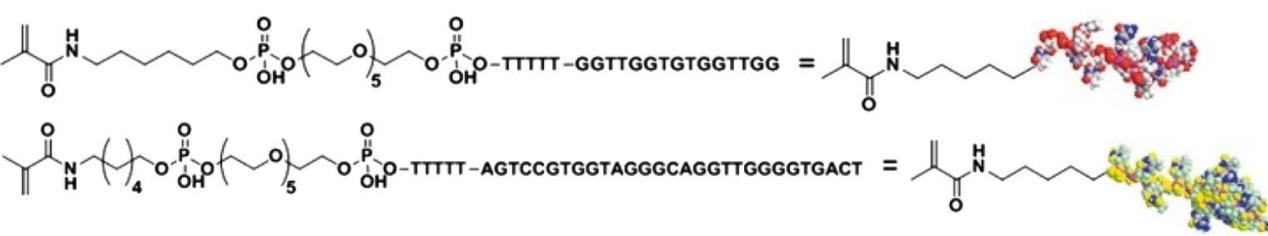

b

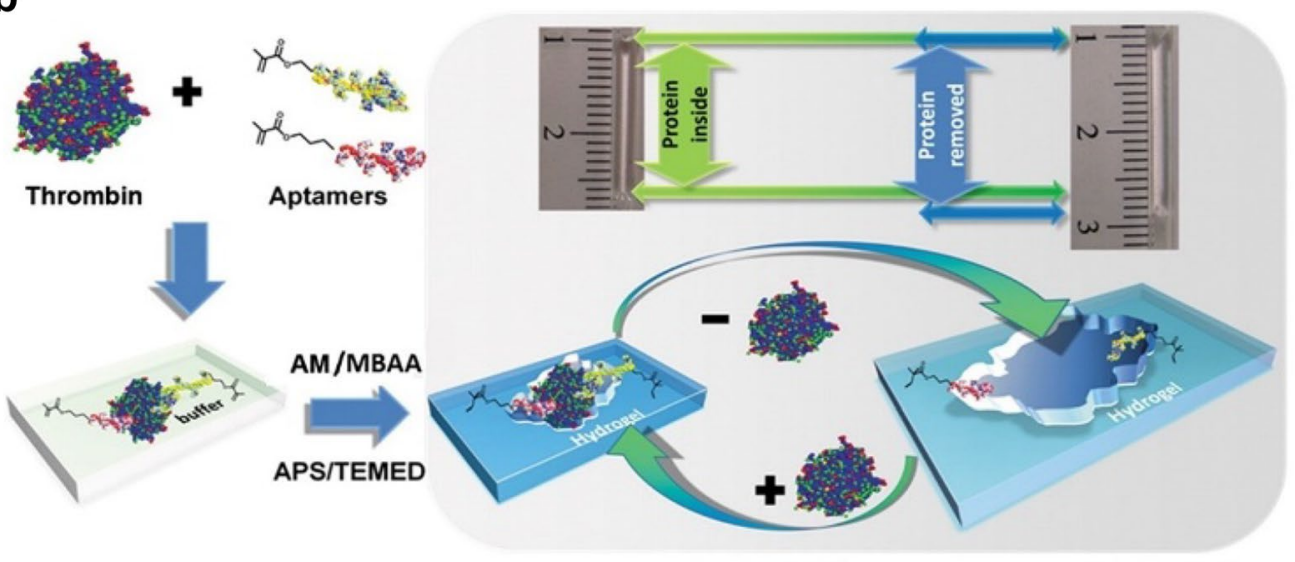

C

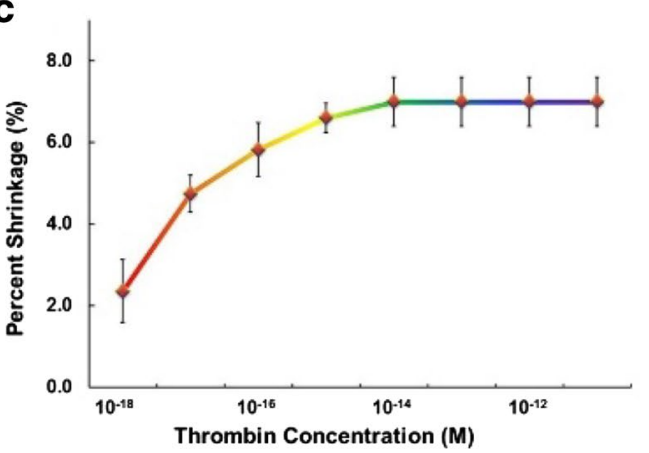

d

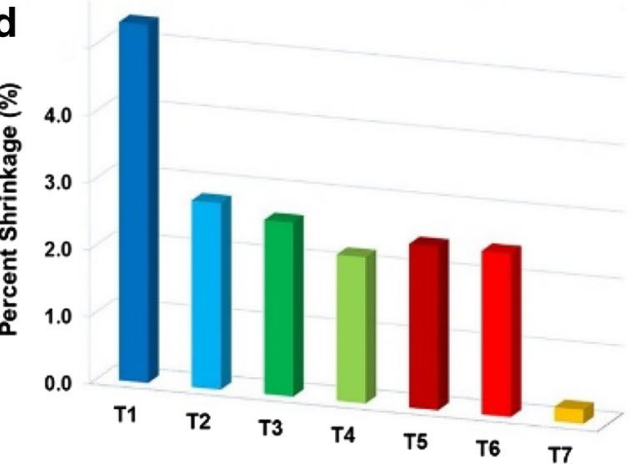

Fig. 8 a The polymerizable aptamers. b Shematic illustration of the imprinted hydrogels with protein-responsive volume change. c The thrombin-dependent shrinkage of the hydrogels. $\mathbf{d}$ The protein-selective hydrogel shrinkage. Reproduced from Ref. [100] with permission from 2013 American Chemical Society 
Imaginably, with the development of polymer chemistry and material science, dynamic MIPs with various responsiveness could be achieved by means of future smart polymers. Similarly, this feature is also suitable for the synthetic receptors based on affinity screening strategy. For example, a temperature-responsive PNIPAAmbased nanoparticle with high affinity towards lysozyme was synthesized via affinity screening strategy [101]. The dynamic property of nanoparticles is capable of temperature-responsive "catch-and-release" of the target protein. However, everything is "a two-sided coin". We have to confess that, although this kind of synthetic receptors are versatile in molecular recognition (i.e., towards almost any target molecules), some special functions like selfhealing and elastomeric properties seem to be impossible, considering the irreversible crosslinking network is the prerequisite to synthesize MIPs or the affinityscreening-based receptors.

\section{Enzyme-like materials}

\section{Supramolecular catalysis}

Inspired by enzymatic system, chemists have developed the field of supramolecular catalysis by utilizing noncovalent interactions to accelerate rate of reaction and/ or allow high selective reactions to occur [51, 54]. Very recently, Leigh's laboratory has reported that knotting molecules can be used to reduce the degrees of freedom of flexible chains, showing thermo-dynamically inaccessible functional conformations. The preorganization of the molecular knots was first formed by metal-organic coordination, which further gave rise to the finally pentafoil knots by in situ covalently linking end groups of each ligand strand. The obtained knot architectures can promote the cleavage of a carbon-halogen bond, bringing advantages of chemo- and stereo-selectivity in chemical reactions traditionally promoted by silver salts (Fig. 9) [102]. Apart from the catalysis based on the knotting molecules, the same group has also introduced a [2] rotaxane to exploit asymmetric catalysis. Under the function of a bulky group in the middle of the thread in the rotaxane, a benzylic amide macrocycle shuttling between different sides of a prochiral center was prevented and generated a chiral space that is suitable for asymmetric catalysis [103].

Generally, a reaction can be catalysed by catalysts that stabilize the transition state of the reaction. Otto's group has discovered that the transition state of a reaction can be associated with and stabilized by a dynamic molecular network. Interestingly after the reaction was completed, the catalyst was disassembled, opening up new opportunities for controlling catalysis in synthetic chemical systems [104]. Tiefenbacher et al. successfully used a supramolecular pocket to mimic cyclase

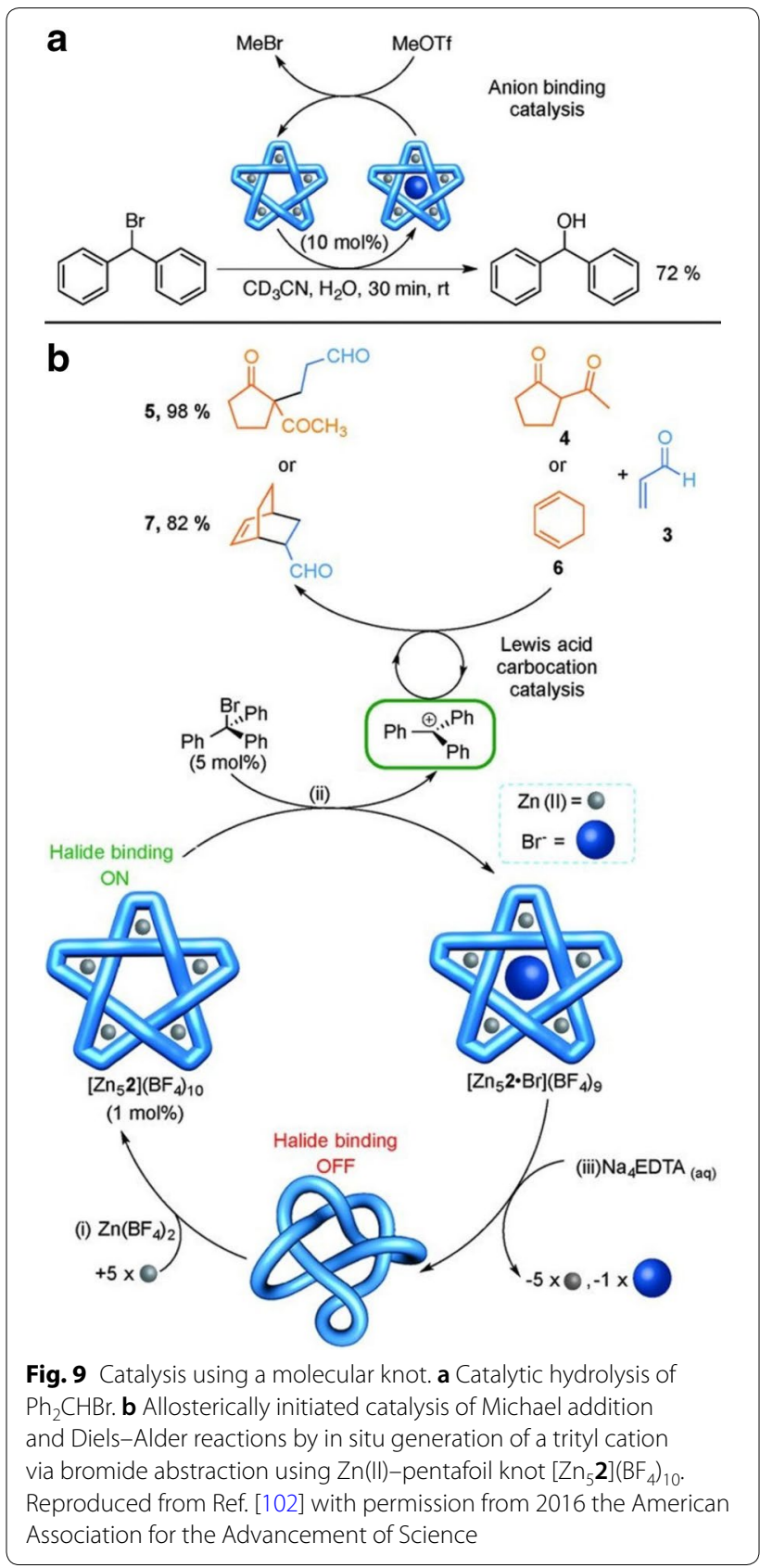

enzymes and achieved a catalytic non-stop tail-to-head terpene with geranyl acetate as the substrate. Remarkably they demonstrated that the direct isomerization of a geranyl cation to the cisoid isomer, which was previously considered infeasible [105]. Subsequently, a detailed mechanism was elucidated. The rate determining step of the cyclization of geranyl acetate was the cleavage of the leaving group, but the reaction needs trace amounts of acid as cocatalyst. A series of control experiments were performed to reveal that the 
catalytic activity was resulted from a synergistic interplay between the supramolecular capsule and the acid traces is required for catalytic activity [106].

These latest examples have clearly emphasised the trend in supramolecular catalysis achieved from the cooperation of molecules in multicomponent systems. The catalytic function of the complex systems can be a combined effect produced by the related components.

\section{Molecularly imprinted catalysis}

MIPs with specific nanosized cavities by means of template directed synthesis can also be utilized as enzymelike catalysts. Compared to biological macromolecules, MIPs are suitable for a wider range of conditions because of the inherent thermal and chemical stability [107]. Generally, a transition state analogue (TSA) of the reactions were used as template to produce polymers with catalytic function. The imprinted sites were utilized either as a supported reagent or to provide temporary functional group protection. Until now, success has been obtained with the applications of MIPs in various reactions, such as dehydrofluorination, sigmatropic shift reaction, stereoselective hydrolysis, and the Diels-Alder and aldol reactions [108-111].
Wulff and co-workers have made enormous contributions on the development of MIPs for catalysis-related applications. Amidinium functional groups were oriented in imprinted cavities which acted as anchors for binding the tetrahedral transition states of basic ester or carbonate hydrolysis to imitate the to the catalytic role of guanidinium moieties in certain catalytic antibodies. Later on, by the same group, a $\mathrm{Zn}^{2+}$ centre was also oriented in a TSA imprinted cavity in a similar way to the active site in carboxypeptidase A (Fig. 10) [112]. The obtained MIPs catalysts are more efficient than catalytic antibodies. In another study, Zhang et al. demonstrated the utilization of MIPs as effective nanoreactors for Huisgen 1,3-dipolar cycloaddition of azides and alkynes [113]. Recently, Shen et al. also reported a MIP microgel stabilized Pickering emulsions (PEs) with ability to catalyze the formation of disulfide bonds in peptides at the O/W interface [114]. Gu et al. prepared a metronidazole-imprinted polymer with nitroreductase-like activity. Accordingly, the imprinted polymer, having both catalysis centers and recognition sites, exhibited enhanced electrocatalytic activity and selectivity [111]. Most recently, Shaabani et al. designed a MIP nano-reactor via miniemulsion polymerization, and the catalytic activity was investigated in
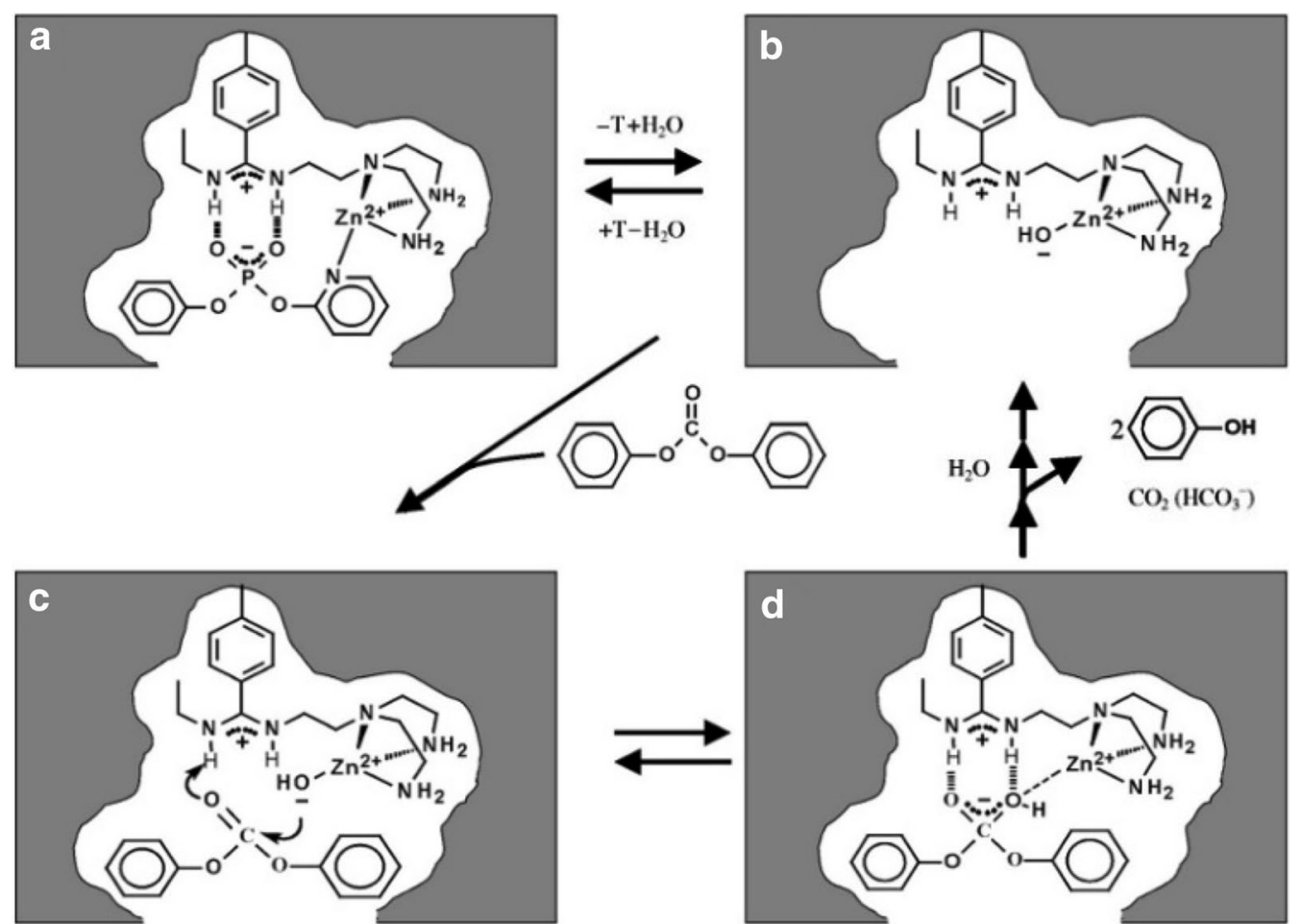

Fig. 10 Schematic representation of $\mathbf{a}$ the molecular imprinting with template $(T)$ and monomer in the presence of $\mathrm{Zn}^{2+}$, $\mathbf{b}$ removal of the template, and c, d catalysis. Reproduced from Ref. [112] with permission from 2004 John Wiley and Sons 
multicomponent reaction transformations for the first time. They demonstrated the applicability of MIP nanoreactors in a one-pot expeditious synthesis of tri- and tetra-substituted imidazole derivatives via pseudo-fourand four-component reactions with excellent yields and purities [115].

It is worth to mention that dynamic MIPs with switchable molecular recognition could exhibit tunable catalytic activity. For example, by using thermo-responsive PNIPAAm, Li et al. designed an 'On/off'-switchable catalysis based on $p$-nitrophenyl phosphate-imprinted networks [116]. The thermo-responsive enzyme-like MIPs showed vigorous catalysis for the hydrolysis of p-nitrophenyl acetate at $20^{\circ} \mathrm{C}$ but poor catalysis at $40{ }^{\circ} \mathrm{C}$. In addition, MIPs could also be indirectly used for catalysis. Very recently, Liu's group successfully engineered MIP binding pockets on inorganic nanozymes for enhancing the oxidation of $\operatorname{TMB}\left(3,3^{\prime}, 5,5^{\prime}\right.$-tetramethylbenzidine) (Fig. 11) [117, 118]. With improved binding specificity, the MIP-engineered nanozymes could reach $\sim 100$ fold of activity and specificity for oxidation of TMB with $\mathrm{H}_{2} \mathrm{O}_{2}$ [118]. This result indicated that, with the assistant of receptor-like specificity in MIPs, the functional enzyme mimicking aspect of nanozymes was greatly developed, and such hybrid materials might find applications in biosensor development, separation, environmental remediation, and drug delivery.

Overall, compared to supramolecular catalysis, molecular imprinting provides the possibility of generating more complicated active sites with a high similarity to

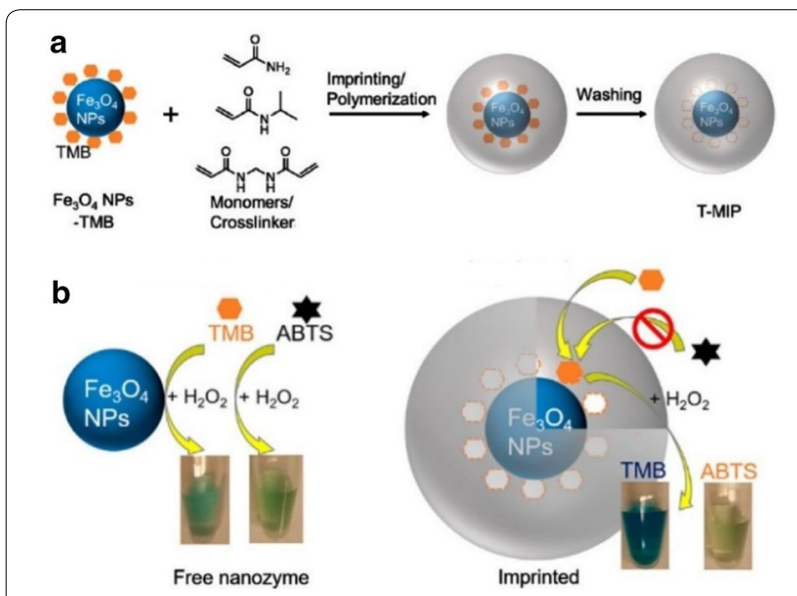

Fig. 11 a A scheme of imprinting TMB

(3,3',5,5'-Tetramethylbenzidine) on $\mathrm{Fe}_{3} \mathrm{O}_{4}$ nanoparticles. $\mathbf{b}$ The activity and specificity of $\mathrm{Fe}_{3} \mathrm{O}_{4}$ nanoparticles and TMB-MIP nanogels for oxidation of TMB and ABTS with $\mathrm{H}_{2} \mathrm{O}_{2}$. The activity of molecular imprinted layer on Inorganic nanozymes shows hundred-fold increase as compared to the $\mathrm{Fe}_{3} \mathrm{O}_{4}$ nanoparticles. Reproduced from Ref. [119] with permission from 2017 American Chemical Society natural systems. The high specificity and strong stability endowed the MIPs an excellent material to mimic the active site of natural enzymes. In fact, non-template synthetic nanoparticles can also exhibit as enzyme-like catalysis through the affinity screening strategy. Wong et al. reported a Poly( $N$-isopropylacrylamide) nanoparticles as an artificial amidase [119]. The mechanism is similar to a MIP-based catalyst. Considering the feasibility of biomolecular recognition, these new polymeric catalysts are very promising for broad application in many fields, in particular, mimicking the enzyme-like catalysis in biological systems.

\section{Bio-recognition materials}

The bio-recognition materials, as the name implies, are the functional materials with affinity to biomolecules. With this property in chemically designed materials, similar functions in biological systems can be mimicked even beyond [120]. Currently, synthetic receptors that can recognize biomolecules mainly concentrate on molecular imprinted polymers and the affinity-screened nanoparticles. Supramolecular assembled receptors based on DCC mainly focus on protein inhibitors discovery $[50,51]$ and they are rarely reported for other bio-recognition, $[6$, $121,122]$ probably due to the requirements of precise chemical structures/functions for molecular assembly and the extremely complicated and dynamic structures of biomolecules. Given this, here we only highlighted some important and emerging applications of bio-recognition based on the MIPs and affinity-screened nanoparticles, for example, bio-separation, controlled release, cell targeting or capture. In view of their great importance in the physiology and pathological processes, small biomolecules like glycans and lipids, macromolecular peptides and proteins are the most popular targets for the synthetic receptors chemically designed by molecular imprinting and affinity screening.

\section{Toxin neutralization}

The pioneering application of MIPs-based molecular recognitions in bio-separation is employing MIPs as the "plastic antibody" for toxin neutralization both in vitro and in vivo $[42,123]$. Shea and co-workers prepared a type of protein-sized polymer nanoparticles (NPs), with a binding affinity and selectivity comparable to those of natural antibodies, by combining a functional monomer optimization strategy with molecular-imprinting nanoparticle synthesis (Fig. 12). The molecularly imprinted NPs with the size comparable to protein clusters have specific binding affinity for the bee toxin melittin. In vivo studies showed that the NPs is not toxicity. More importantly, in vivo imaging of the polymer nanoparticles showed that the NPs accelerated clearance of the toxin 


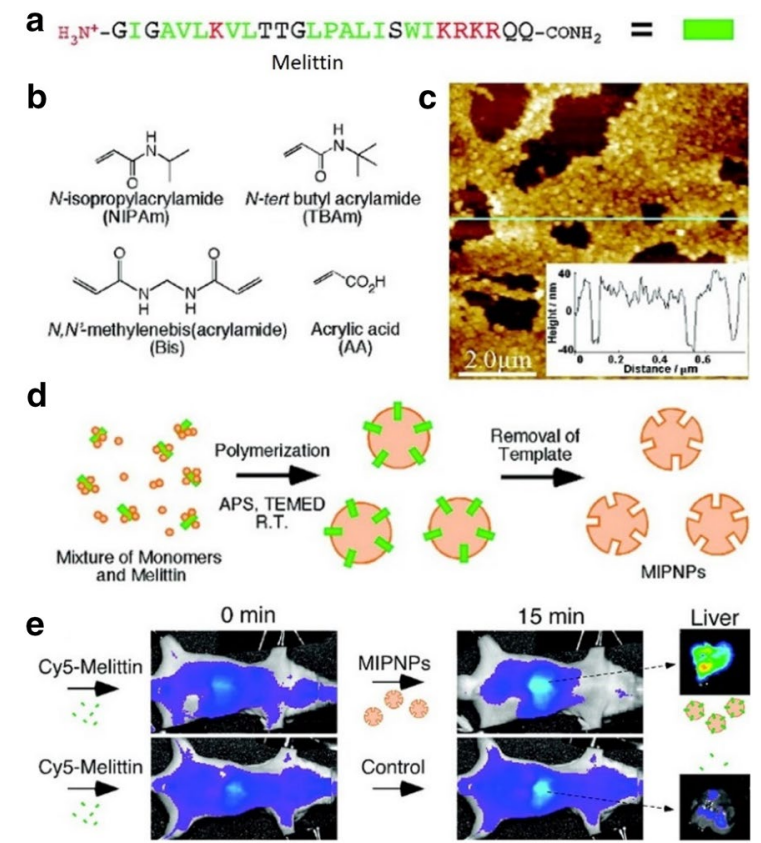

Fig. 12 Preparation and characterization of MIP nanoparticles. a Amino acid sequence of target peptide Melittin. b Monomers used for nanoparticle synthesis. c Solution phase AFM images of MIP nanoparticles. $\mathbf{d}$ Schematic of the preparation of MIP nanoparticles. e Biodistribution of melittin and MIP nanoparticles after intravenous injection of Cy5-melittin. MIP nanoparticles was injected $20 \mathrm{~s}$ after the injection of melittin. Reproduced from Ref. [124] with permission from 2010 American Chemical Society

peptide from blood where they accumulate in the liver. This result verified that the nanoparticles can recognize and neutralize toxin in vivo, thus facilitating the diminishing of mortality and peripheral toxic symptoms in the melittin-injected mice. Clearly, MIPs-based receptors with bio-molecular recognition properties offer potential for neutralizing a wide range of toxic biomacromolecules in vivo.

Worthy of mentioning is that, without an imprinting process, the same group also employed the affinity screening strategy to select nanoparticle candidates with high affinity and selectivity to different peptide toxins, enzymes, and other functional proteins as well as to specific domains of large proteins [44]. For example, the groups recently reported a synthetic polymer nanoparticle (NP) with broad-spectrum sequestration and neutralization of venomous biomacromolecules [124]. The optimized NP exhibited low cytotoxity and showed substantially long dissociation rates from the human platelet alloantigen (PLA2), suggesting that the NP may show efficacy as an in vivo venom sequestrant and may serve as a generalized lipid-mediated toxin sequestrant by extracting toxic matter from the bloodstream.

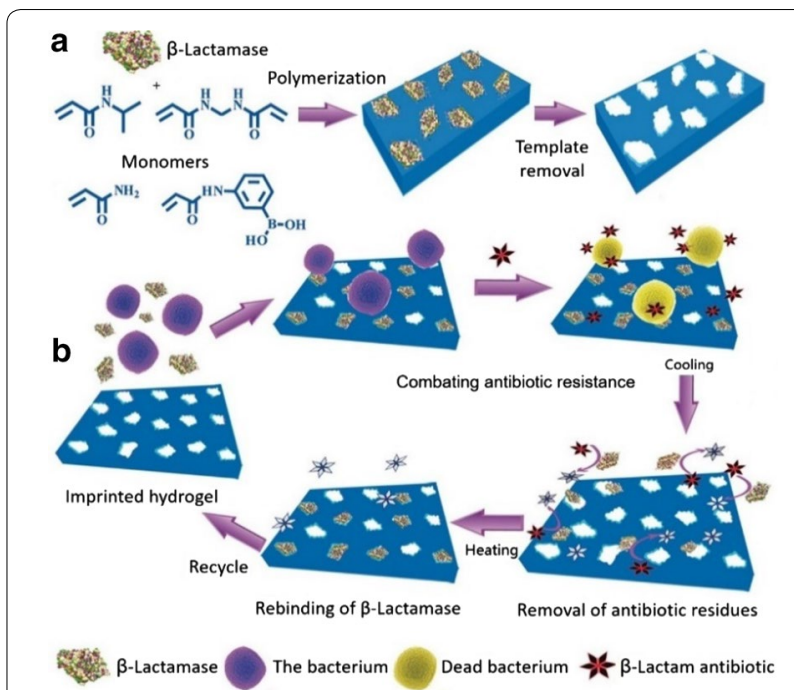

Fig. 13 a Fabrication of a temperature-responsive imprinted hydrogel with $\beta$-lactamase as the template. $\mathbf{b}$ Bacteria could express $\beta$-lactamases to hydrolyze $\beta$-lactam antibiotics. The imprinted hydrogel bound $\beta$-lactamase and protected antibiotics from enzymatic degradation. After bactericidal treatment, the $\beta$-lactamase trapped in the hydrogel was released by a temperature stimulus and could then degrade antibiotic residues. The residual $\beta$-lactamase in solution could be rebound by the IP hydrogel to decrease their health risk. Reproduced from Ref. [126] with permission from 2016 John Wiley and Sons

In addition, similar concept has also been applied to weaken the multi-resistance of bacterial by extracting an antibiotic enzyme, $\beta$-lactamase, from bacterial secretion (Fig. 13) [125]. In their work, Li et al. prepared an imprinted hydrogel with thermo-responsive molecular recognition towards $\beta$-lactamase. As known, $\beta$-lactamase can deactivate antibiotics, thus providing multi-resistance of bacteria to $\beta$-lactam antibiotics. The thermoresponsive $\beta$-lactamase-imprinted hydrogel could initially trap $\beta$-lactamase excreted by the drug-resistant bacteria, thus making the bacteria sensitive to antibiotics and improving antibacterial activity. Thus, the imprinted hydrogel could act as an adjuvant to enhance the efficacy of antibiotics against drug-resistant bacteria. Moreover, the "imprinted sites" on the hydrogel could be reversibly abolished with a temperature stimulus, which resulted in the reactivation of $\beta$-lactamase to degrade antibiotic residues. The authors also demonstrated the success of such antibacterial design to treat wound infection, indicating the promising of this MIP hydrogel for efficient antibiotic therapy.

\section{Protein crystallization}

Another important application of MIPs with biomolecular recognition is their ability to assist protein crystallization. As known, MIPs are made to contain cavities 
capable of rebinding protein. Therefore, the fingerprint of the protein created on the polymer allows it to serve as an ideal template for crystal formation. Saridakis et al. demonstrated that MIPs can facilitate the formation of large single protein crystals at metastable conditions for seven proteins [126]. This is due to the recognition of proteins by the cavities, which would concentrate the target proteins near the interface and lead to protein crystallization. This initiative research implied that MIPs could act as nucleation-inducing substrates by harnessing the target proteins themselves as templates, thus showing the promise to significantly accelerate the discovery of new protein crystal structures.

\section{Protein protection and refolding}

According to the protein affinity of synthetic receptors, researchers in this field considered to employ them to control the functions or structures of target proteins. Initially, synthetic receptors were only applied to inhibit enzyme activity, by virtue of the strong enzyme binding affinity [127]. Recently, Shea's group reported a new concept by applying a thermally responsive PNIPAm-based copolymer nanoparticle hydrogel with good protein affinity for protection of target proteins from thermal stress (Fig. 14) [128]. The protein-binding nanoparticles, prepared by affinity screening strategy hydrogels, could bind and protect a target enzyme from irreversible activity loss upon exposure to heat but "autonomously" release the enzyme upon subsequent cooling of the solution. The results showed that, in the presence of the screened nanoparticle hydrogels, lysozyme could retain over $80 \%$ of its activity after it had been heated at $85{ }^{\circ} \mathrm{C}$ for $30 \mathrm{~min}$. The authors hypothesized that the nanoparticles form a complex with lysozyme due to the high affinity, which could prevent protein aggregation at elevated temperature, thereby mimicking the action of a "passive aggregation inhibitor" type of heat shock protein. This finding demonstrated the promise of this approach for improving the thermal tolerance of proteins. Moreover, such material design concept may be applied for the refolding of chemically denatured proteins and facilitating the protein production by a cell-free protein synthesis system.

A recent report by Nakamoto et al. indeed indicated the potential of a protein-affinity nanoparticles for refolding denatured protein. The study demonstrated that affinity-screened polymeric nanoparticles, prepared by copolymerizing optimized combinations and populations of functional monomers, were capable of facilitating resolubilization and refolding of an aggregated protein, lysozyme [129]. The authors revealed that the facilitation of resolubilization and refolding of aggregated lysozyme is driven by a strong affinity of nanoparticles to denatured lysozyme as well as a relatively weak affinity with native lysozyme. After centrifugal ultrafiltration, the refolded lysozyme showed native conformation and enzymatic activity. Moreover, the synthetic nanoparticles showed excellent productivity on protein refolding, and more than $10 \mathrm{~g}$ of aggregated protein can be efficiently

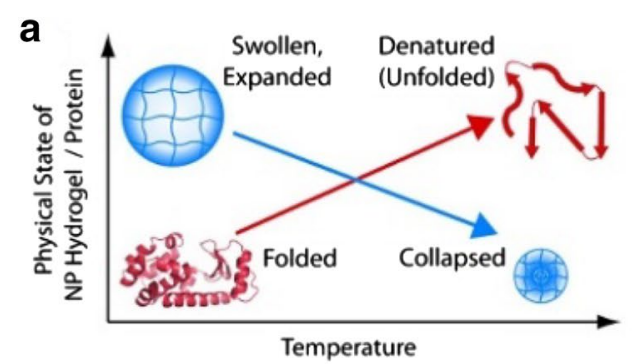

C

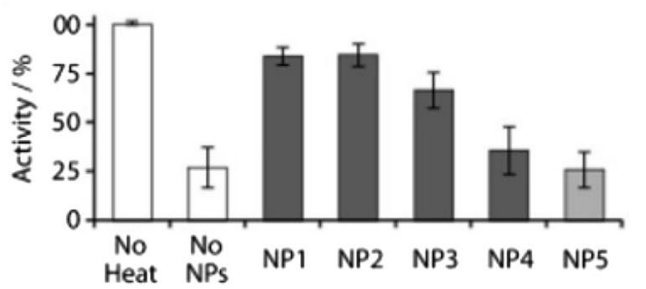

b

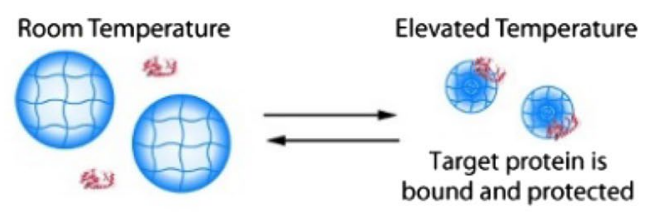

d

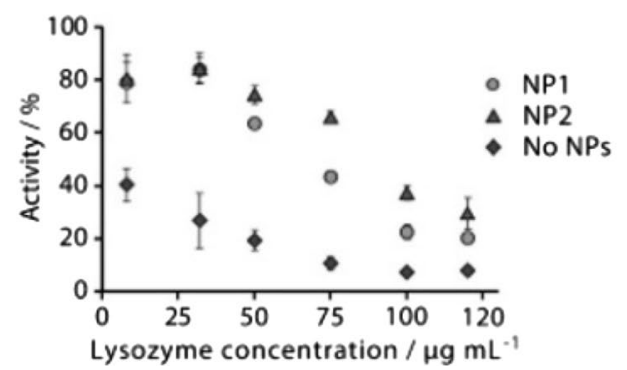

Fig. 14 a Comparison of the heat response of a protein (red) anda PNIPAm-based polymer nanoparticle (blue). Proteins denature (unfold) in response to heat, whereas the nanoparticles contract into a collapsed state. b Illustration of the "auto-nomous affinity switching" property of nanoparticels. c Effect of different polymer nanoparticles $\left(2.0 \mathrm{mgmL}^{-1}\right)$ on the activity of lysozyme after heating. $\mathbf{d}$ Effect of NP1 and NP2 (2.0 $\mathrm{mg} \mathrm{mL}^{-1}$ ) on the activity of different concentrations of lysozyme after heating. Reproduced from Ref. [129] with permission from 2014 John Wiley and Sons 
refolded by only $1 \mathrm{~g}$ of the nanoparticles. These results suggest the great potential of synthetic receptors as artificial chaperones with high facilitating activity for nature biomolecules.

\section{Bioimaging}

Nanomedicine is a rapidly growing field, in particular, in medical oncology, $[66,130,131]$ MIPs with affinity towards tumor-related biomarkers will facilitate targeted diagnosis and therapy, e.g., cancer targeting and drug delivery $[132,133]$. In case of combination with photoelectric materials, MIPs could be used for bioimaging. Cancer targeting for MIPs was usually conducted according to the recognition of specific glycans on the tumor cell surface, such as glucuronic acid, sialic acid (SA), fucose (Fuc), mannose (Man), and so on [134-138]. Liu and co-workers reported SA-imprinted nanoparticles based on $p$-aminothiophenol (PATP, a Raman reporter)functionalized silver nanoparticles (AgNPs) for surface enhanced Raman scattering (SERS) imaging of cancer cells [134] (Fig. 15). The specific interactions between SA and polymer matrix were enhanced by the phenylboronic acid functional groups [64, 139]. After surface molecular imprinting, the SERS nanoparticles were endowed with high specificity toward SA, a cancer biomarker that is overexpressed on several cancer cell lines. Therefore, the

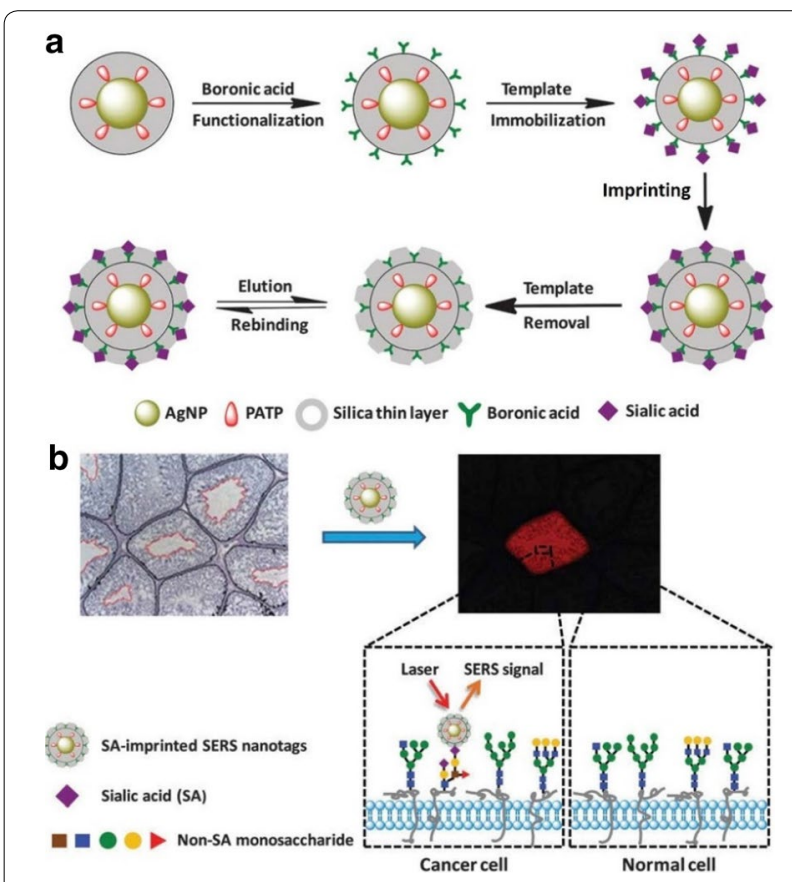

Fig. 15 a Schematic of the synthesis route of SA-imprinted $\mathrm{Ag@SiO}_{2}$ SERS nanoparticles. $\mathbf{b}$ Schematic of SERS imaging of cancer cells and tissues via SA-imprinted nanoparticles. Reproduced from Ref. [135] with permission from 2015 Royal Society of Chemistry
SA-imprinted SERS nanoparticles were able to differentiate cancer cells from normal cells and subsequently visualizing them $[135,136]$.

Recently, researchers in the field of MIPs are consciously shifting their attention to tumor-related biomacromolecules. A small but growing body of evidence has suggested that vascular endothelial growth factor (VEGF) is overexpressed in various cancers cells, such as gastrointestinal, breast, colorectal, etc [140, 141]. Therefore, targeting of VEGF by artificial antibody has been proposed for tumor cell imaging. Very recently, Cecchini et al. prepared human VEGF (hVEGF) imprinted nanoparticles by solid-phase synthesis and coupled to quantum dots (QDs) to enable subsequently fluorescent imaging in vivo (Fig. 16) [142]. The VEGF-MIPs could specifically target hVEGF and homing towards the tumor mass in xenotransplantation of human malignant melanoma cells in zebrafish embryos. This sample indicated that nano-MIPs are promising materials, which can be considered for advancing molecular oncological research, in particular when antibodies are less desirable due to their immunogenicity or long production time. Furthermore, targeting VEGF could be also achieved by affinity screening strategy. Koide et al. prepared a polymer nanoparticle with engineered affinity for VEGF by incorporating a trisulfated $N$-acetylglucosamine monomer, $N$-tert-butylacrylamide, in a crosslinked NIPAm copolymer nanoparticles [143]. The result revealed that synthetic nanoparticles can be engineered to bind to and interfere with the signalling protein (VEGF165) by targeting specific domains of the protein, also implying the great potential for targeted cell imaging.

In general, molecular imprinted or affinity screened synthetic receptors have now aroused increasing interest in bio-imaging. The flexibility in the selection of targeted molecules makes these synthetic receptors as promising candidates for the visualization of various tissues and cells. However, significant efforts are still urgently required to further overcome some drawbacks, such as rapid photo-bleaching, potentially toxic, and the relatively low targeting efficiency in vivo.

\section{Cancer inhibition}

Taking the cell imaging one step further, researchers also try to employ tunor-cell-targeted nano-MIPs for enhanced cancer therapy. Recently, Zhang et al. reported an imprinted nanoparticle that was found to bind strongly to a membrane protein $\mathrm{p} 32$, which was overexpressed on the surface of a variety of tumour cells (Fig. 17) [144]. Cell targeting ability was first confirmed by the higher uptake of imprinted nanoparticles compared to control nanoparticles by p32-positive cancer cells. In vivo study showed that the nanoparticles encapsulating a fluorophore dye 


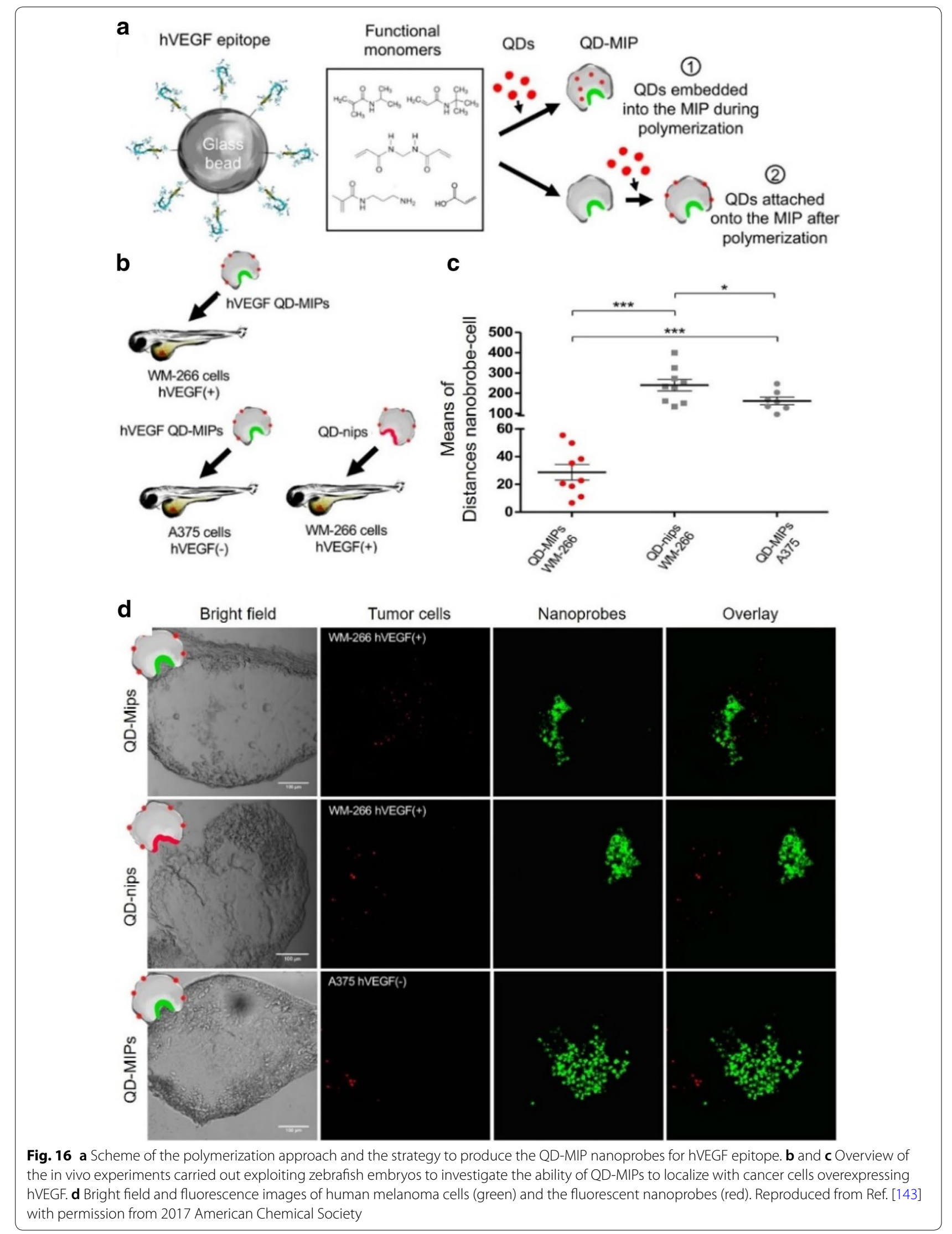




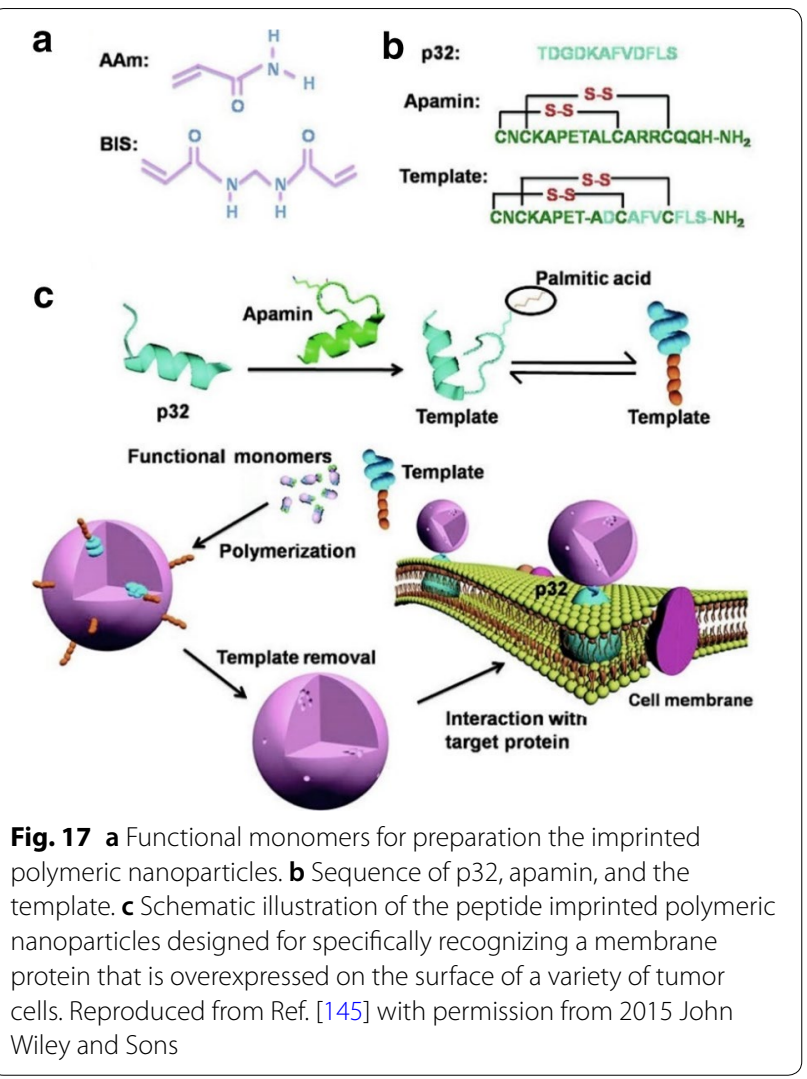

(methylene blue) led to considerably higher accumulation of imprinted than of non-imprinted nanoparticles in a mouse xenograft tumour, implying the potential to mediate targeted drug delivery for therapy. The same group further prepared an imprinted nanoparticles that could recognize the transmembrane domain of target receptors [145]. A transmembrane helical peptide was used as a template, and 2,2,2-trifluoroethanol (TFE) was added to maintain a stable conformation for the peptide template. The MIP nanoparticles could enhance the cellular uptake and permeability in target tissues for tumor-targeted drug delivery. Another group also designed an imprinted gold nanorod (AuNR) for targeted photothermal cancer therapy [146]. Sialic acid (SA) was employed as the template for the preparation of MIPs. The SA-imprinted AuNR exhibited good cancer cell targeting selectivity as well as high photothermal effect. Moreover, the targeted plasmonic nanomaterial was able to selectively kill tumor cells without damaging the surrounding healthy tissue. The advantage of using MIP nanoparticles as drug-delivery vehicles for targeted chemo- or photodynamic cancer therapy is the flexible selectivity. It can target not only the fully exposed transmembrane proteins or monosaccharide on the tumor cell membranes, but also the partially exposed transmembrane proteins via specific three-dimensional shape recognition.

\section{Cell capture and release}

Dynamic interactions between natural receptors at the cell membrane and ligands at the extracellular matrix (ECM) are crucial in cellular processes [147]. Materials capable to dynamic display bioactive ligands and modulating specific cell-biomaterial interactions have attracted increasing attentions in both fundamental cell biology, medical diagnostics and tissue engineering [148-150]. Molecular recognition is commonly dynamic process, implying its potential for reversible display of bioligands on materials and control of cell-materials interactions by using the biomolecular affinity. Pan et al. reported a PNIPAm-based MIP hydrogel for reversible cell adhesion [97] that relied on thermoresponsive affinity toward peptide RGDS, a cell adhesive factor for the cell membrane integrin $\alpha_{\mathrm{v}} \beta_{3}$ [151]. In their design, molecular imprinting methodology was employed to create the molecular recognition sites for targeted RGDS peptide onto a thermo-responsive hydrogel, which was innovatively used as a highly efficient cell culture substrate for harvesting cell sheets (Fig. 18a). The thermo-responsive molecular recognition sites on MIP hydrogel could not only promote cell adhesion during cell culture but also facilitate cell detachment during cell sheet harvesting process. This is the first sample that successful use of a synthetic receptor for modulating cell-material interactions. However, it is worth mentioning that the poor accessibility of the bound RGD peptides embedding in recognition sites greatly limited efficient bioactivity presentation on the material interfaces. Recently, Pan et al. further reported an epitope imprinting process [152] for dynamic binding of bioactive ligands on the material interface (Fig. 18b) [153]. The authors used a terminal short peptide sequence (epitope peptide) of an RGD-based long peptide as the template during the imprinting process. After peptide binding on the MIP layer, the epitope peptide could act as a reversible anchor of the RGD peptide leaving the latter exposed for interacting with the cell surface integrin receptors. More important, addition of the epitope peptide to the system could trigger the release of bound RGD peptides through competitively molecular exchange. The results showed that the epitope imprinted layer enabled reversible presentation of cell adhesive peptide and subsequently cell adhesion on the surface. Such a molecularly tunable dynamic system based on the molecular recognition of MIPs may unlock new applications in in situ cell biology, diagnostics and regenerative medicine.

\section{Conclusions and outlook}

As one of the most fundamental events capable of inducing the further advanced reactions, molecular recognition has been well developed into a mature field in which exploring synthetic receptor becomes a primary objective. Many novel strategies have been initiated to target at 
a

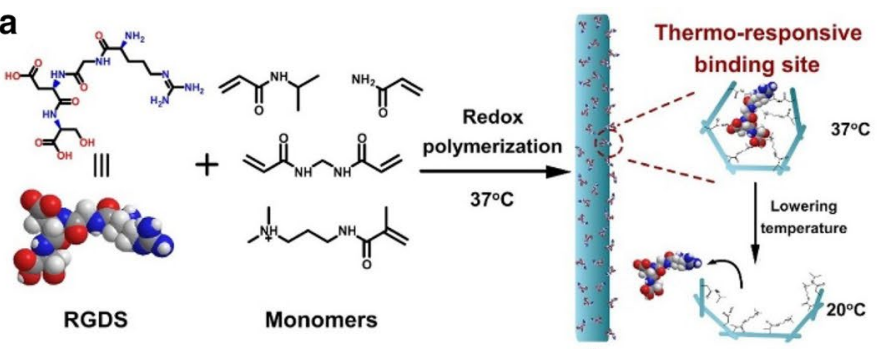

RGDS release and rapid cell sheet detachment

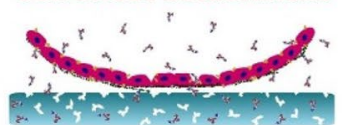

b

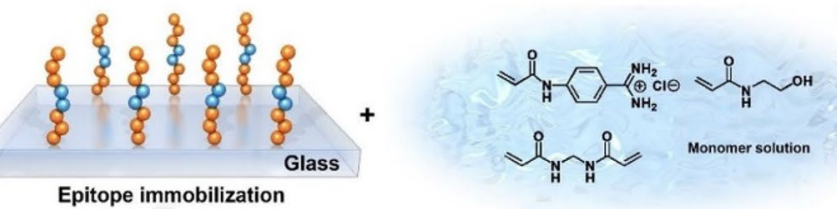

Epitope immobilization

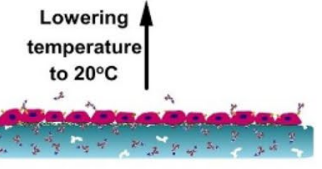

Cell sheet formation

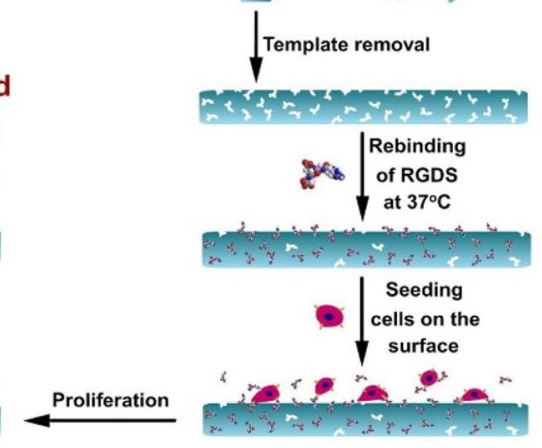

Cell recognition and adhesion

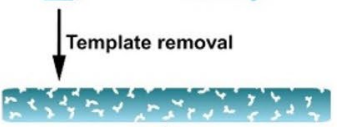

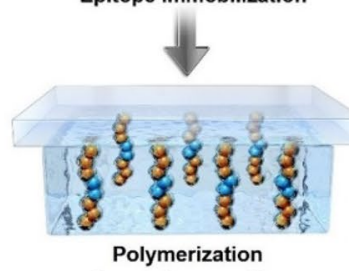

Polymerization
Cover glass peeling

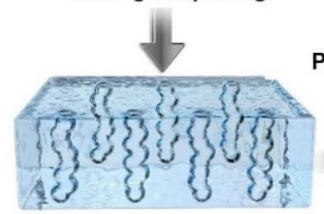

Peptide bearing RGD

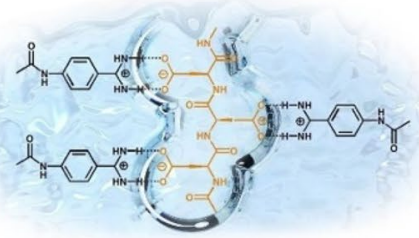

corpood 88

Rebinding

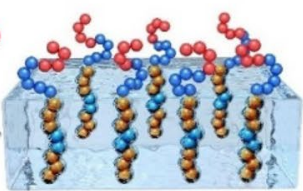

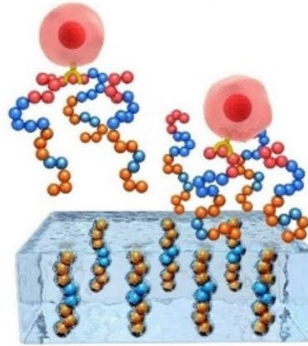

Cell release

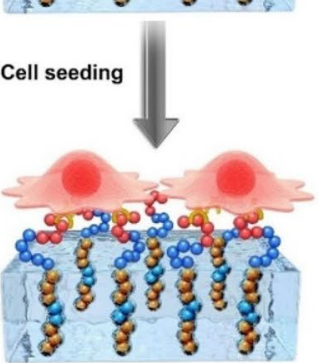

Cell adhesion

Fig. 18 a Strategy to introduce the RGDS peptide on a thermo-responsive cell culture substrate for reversible cell adhesion and harvesting of a cell sheet by means of molecular imprinting, $\mathbf{b}$ Schematic illustration of the epitope imprinted biointerface for dynamic cell adhesion. Reproduced from Refs. $[97,153]$ with permission from John Wiley and Sons 
effective receptors that can selectively associate with specific guest molecules. Thanks to the progress in computer science, rational design is helpful to predict host molecules suitable for metal ions, anions and even organic guest molecules. Molecular imprinting endows polymers with specific molecular recognition sites complementary to target molecules and has shown to be the most successful technique regards to binding efficiency and selectivity. However, template molecules are requisite for the imprinting process, which made the MIPs-based recognition towards complicated biomolecules into trouble. Fortunately, affinity screening Affinity screening of synthetic polymers with different chemical compositions and functional groups provide a promising strategy for massively exploiting new receptors for bio-recognition. This simple and feasible strategy withour using template molecules is a great supplement for molecular imprinting. By merging the dynamic covalent chemistry with noncovalent interactions, DCC has become a powerful tool for synthesizing receptors directed by association at a systems level. Even so, DCC is rarely reported for biomolecular recognition. This is probably due to the requirements of precise chemical structures/functions for molecular assembly, which become extremely complicated in case of dynamic structures of biomolecules.

The ultimate goal of molecular recognition is to implement artificial receptors into a range of novel applications. We have given a brief summary of the recent applications of molecular recognition in exploring novel smart materials, catalysts and bioimaging of disease for diagnosis and therapy. However, the ubiquitousness of molecular recognition is far beyond these developments, but also far beyond the boarder that we can envision. It is not possible to predict with any certainty where the field should go in the following years. We only can suggest the research that can be further investigated from the recent progress. For example, compared with the number of allosteric receptors in nature, the man-made ones are much less investigated. It is an apt time to explore dynamic molecular recognition in a larger context with self-assembly, which may give rise to versatile complex materials. Combination of molecular imprinting with more sensitive sensor platforms and microfluidic devices is expected to yield more attractive commercial development and applications. Moreover, incorporating high-throughput synthesis and new analysis technique in the molecular imprinting and affinity screening strategy as well as the combination with the exploitation of novel functional monomers are conductive to rapid generation of inexpensive and uniquely bio-selective receptor-like nanomaterials that would lead to unpredictable advances in biotechnology, in particular, the applicability in vivo. For man-made molecular recognition, this is the fort must be conquered. During the past decades, enormous amounts of supramolecular architectures have formed via molecular recognition at equilibrium. However, their functions and the development in practical applications are always overlooked. Leigh et al. has been starting to show the significance of the knotting structure for kinetic control over a chemical reaction, which will lead a trend to this direction. There is no doubt that there also have many other directions into which molecular recognition can devote. We believe that the fundamental event of molecular recognition and the increasing attention on synthetic receptors will play an increasingly key role in bridging the gap between molecular science and advanced functional materials.

\section{Abbreviations \\ DCC: dynamic combinatorial chemistry; MI: molecular imprinting; MIPs: molecularly imprinted polymers; SWCNTs: single-walled carbon nanotubes; $\beta C D$ : $\beta$-cyclodextrin; ATP: adenosine-5'-triphosphate; PNIPAm: Poly(N-isopro- pylacrylamide); LCST: lower critical solution temperature; HSA: human serum albumin; BSA: bovine serum albumin; TSA: transition state analogue; PEs: Pick- ering emulsions; TMB: 3,3',5,5'-tetramethylbenzidine; NPs: nanoparticles; PLA2: human platelet alloantigen; PATP: p-aminothiophenol; SERS: surface enhanced Raman scattering; VEGF: vascular endothelial growth factor; QDs: quantum dots; TFE: 2,2,2-trifluoroethanol; AuNR: gold nanorod.}

\section{Acknowledgements}

Not applicable.

\section{Authors' contributions}

WC, XT and WH contributed equally to this work. WC, XT and WH wrote the manuscript under the guidance of $J L$ and GP. JL, YF and GP designed the manuscript. All authors read and approved the final manuscript.

\section{Funding}

This work was financially supported by the National Key Research and Development Program of China (2019YFA0112000), the National Natural Science Foundation of China (21875092 and 91649204), the Innovation and Entrepreneurship Program of Jiangsu Province, and the "Six Talent Peaks" program of Jiangsu Province (2018-XCL-013). Funding sources played no role in the design of the study, collection, analysis, interpretation of the data or writing the manuscript.

Availability of data and materials

Not applicable.

\section{Competing interests}

The authors declare that they have no competing interests.

\section{Author details}

${ }^{1}$ Institute for Advanced Materials, School of Materials Science and Engineering, Jiangsu University, Zhenjiang 212013, Jiangsu, China. ${ }^{2}$ College of Chemical and Environmental Engineering, Shandong University of Science and Technology, Qingdao 266590, Shandong, China. ${ }^{3}$ Hainan Provincial Key Lab of Fine Chem, Key Laboratory of Advanced Materials of Tropical Island Resources of Ministry of Education, Hainan University, Haikou 570228, China.

Received: 26 April 2019 Accepted: 11 December 2019

Published online: 09 January 2020

\section{References}

1. Frederick KK, Marlow MS, Valentine KG, Wand AJ. Conformational entropy in molecular recognition by proteins. Nature. 2007:448:325-9. 
2. Persch E, Dumele $\mathrm{O}$, Diederich F. Molecular recognition in chemical and biological systems. Angew Chem Int Ed. 2015;54:3290-327.

3. Baron R, McCammon JA. Molecular recognition and ligand association. Annu Rev Phys Chem. 2013;64:151-75.

4. Barton NH, Briggs DE, Eisen JA, Goldstein DB, Patel NH, Evolution of novelty. Evolution. 2007; 695-724.

5. Subrahmanyam S, Piletsky SA, Turner AP. Application of natural receptors in sensors and assays. Anal Chem. 2002;74:3942-51.

6. Mahon CS, Fulton DA. Mimicking nature with synthetic macromolecules capable of recognition. Nat Chem. 2014;6:665-72.

7. Ruigrok VJ, Levisson M, Eppink MH, Smidt H, Van Der Oost J. Alternative affinity tools: more attractive than antibodies? Biochem J. 2011:436(1):1-13.

8. Vlatakis G, Andersson LI, Müller R, Mosbach K. Drug assay using antibody mimics made by molecular imprinting. Nature. 1993;361:645-7.

9. Webber MJ, Appel EA, Meijer E, Langer R. Supramolecular biomaterials. Nat Mater. 2016;15:13-26.

10. Loh XJ. Supramolecular host-guest polymeric materials for biomedical applications. Mater Horiz. 2014;1:185-95.

11. MaX, Zhao Y. Biomedical applications of supramolecular systems based on host-guest interactions. Chem Rev. 2014;115:7794-839.

12. Harada A, Takashima Y, Nakahata M. Supramolecular polymeric materials via cyclodextrin-guest interactions. Acc Chem Res. 2014:47:2128-40.

13. Zhou J, Yu G, Huang F. Supramolecular chemotherapy based on hostguest molecular recognition: a novel strategy in the battle against cancer with a bright future. Rev: Chem. Soc; 2017.

14. Barrow SJ, Kasera S, Rowland MJ, del Barrio J, Scherman OA. Cucurbituril-based molecular recognition. Chem Rev. 2015;115:12320-406.

15. Dong S, Luo Y, Yan X, Zheng B, Ding X, Yu Y, Ma Z, Zhao Q, Huang F. A dual-responsive supramolecular polymer gel formed by crown ether based molecular recognition. Angew Chem. 2011;123:1945-9.

16. Murray J, Kim K, Ogoshi T, Yao W, Gibb BC. The aqueous supramolecular chemistry of cucurbit [n] urils, pillar [n] arenes and deep-cavity cavitands. Chem Soc Rev. 2017;46:2479-96.

17. Schmidt B, Barner-Kowollik C. Dynamic Macromolecular Material Design-The Versatility of Cyclodextrin Based Host/Guest Chemistry. Chem: Angew; 2017.

18. Lehn J-M. Supramolecular chemistry: where from? Where to? Chem Soc Rev. 2017:46:2378-9.

19. Li D, Chen Y, Liu Z. Boronate affinity materials for separation and molecular recognition: structure, properties and applications. Chem Soc Rev. 2015;44:8097-123.

20. Ariga $\mathrm{K}$, Ito $\mathrm{H}$, Hill JP, Tsukube $\mathrm{H}$. Molecular recognition: from solution science to nano/materials technology. Chem Soc Rev. 2012;41:5800-35.

21. Jin Y, Wang Q, Taynton P, Zhang W. Dynamic covalent chemistry approaches toward macrocycles, molecular cages, and polymers. Acc Chem Res. 2014;47:1575-86.

22. Pan J, Chen W, Ma Y, Pan G. Molecularly imprinted polymers as receptor mimics for selective cell recognition. Rev: Chem. Soc; 2018.

23. Wulff G. Molecular imprinting in cross-linked materials with the aid of molecular templates - a way towards artificial antibodies. Angew Chem Int Ed. 1995;34:1812-32.

24. Mosbach K. Molecular imprinting. Trends Biochem Sci. 1994;19:9-14.

25. Hart BR, Shea KJ. Synthetic peptide receptors: molecularly imprinted polymers for the recognition of peptides using peptide - metal interactions. J Am Chem Soc. 2001;123:2072-3.

26. Ma Y, Pan G, Zhang Y, Guo X, Zhang H. Comparative study of the molecularly imprinted polymers prepared by reversible addition-fragmentation chain transfer "bulk" polymerization and traditional radical "bulk" polymerization. J Mol Recognit. 2013;26:240-51.

27. Wang J, Dai J, Xu Y, Dai X, Zhang Y, Shi W, Sellergren B, Pan G. Molecularly imprinted fluorescent test strip for direct, rapid, and visual dopamine detection in tiny amount of biofluid. Small. 2019;15:1803913.

28. Wang J, Qiu H, Shen H, Pan J, Dai X, Yan Y, Pan G, Sellergren B. Molecularly imprinted fluorescent hollow nanoparticles as sensors for rapid and efficient detection $\lambda$-cyhalothrin in environmental water. Biosens Bioelectron. 2016;85:387-94.

29. Haupt K, Mosbach K. Molecularly imprinted polymers and their use in biomimetic sensors. Chem Rev. 2000;100:2495-504.
30. Wan L, Chen Z, Huang C, Shen X. Core-shell molecularly imprinted particles. Trends Anal Chem. 2017;95:110-21.

31. Wuff G, Sarhan A. The use of polymers with enzyme-analogous structures for the resolution of racemate. J Angew Chem Int Ed. 1972;11:341-5.

32. Sellergren B. Imprinted polymers with memory for small molecules, proteins, or crystals. Angew Chem Int Ed. 2000;39:1031-7.

33. Bossi A, Bonini F, Turner A, Piletsky S. Molecularly imprinted polymers for the recognition of proteins: the state of the art. Biosens Bioelectron. 2007;22:1131-7.

34. Pan G, Zhang Y, Ma Y, Li C, Zhang H. Efficient one-pot synthesis of water-compatible molecularly imprinted polymer microspheres by facile RAFT precipitation polymerization. Angew Chem Int Ed. 2011;50:11731-4.

35. Gunasekara RW, Zhao Y. A general method for selective recognition of monosaccharides and oligosaccharides in water. J Am Chem Soc 2017;139:829-35.

36. Liu S, Pan J, Zhu H, Pan G, Qiu F, Meng M, Yao J, Yuan D. Graphene oxide based molecularly imprinted polymers with double recognition abilities: the combination of covalent boronic acid and traditional noncovalent monomers. Chem Eng J. 2016;290:220-31.

37. Li R, Feng Y, Pan G, Liu L. Advances in molecularly imprinting technology for bioanalytical applications. Sensors. 2019;19:177.

38. Chen L, Wang X, Lu W, Wu X, Li J. Molecular imprinting: perspectives and applications. Chem Soc Rev. 2016;45:2137-211.

39. Yin Y, Pan J, Cao J, Ma Y, Pan G, Wu R, Dai X, Meng M, Yan Y. Rationally designed hybrid molecularly imprinted polymer foam for highly efficient $\lambda$-cyhalothrin recognition and uptake via twice imprinting strategy. Chem Eng J. 2016;286:485-96.

40. Rensing S, Arendt M, Springer A, Grawe T, Schrader T. Optimization of a synthetic arginine receptor systematic tuning of noncovalent interactions. J Org Chem. 2001;66(17):5814-21.

41. Whitby LR, Boger DL. Comprehensive peptidomimetic libraries targeting protein-protein interactions. Acc Chem Res. 2012;45:1698-709.

42. Hoshino Y, Haberaecker WW III, Kodama T, Zeng Z, Okahata Y, Shea KJ. Affinity purification of multifunctional polymer nanoparticles. J Am Chem Soc. 2010;132:13648-50.

43. Koch SJ, Renner C, Xie X, Schrader T. Tuning linear copolymers into protein-specific hosts. Angew Chem Int Ed. 2006;45:6352-5.

44. O'Brien J, Shea KJ. Tuning the protein corona of hydrogel nanoparticles: the synthesis of abiotic protein and peptide affinity reagents. Acc Chem Res. 2016:49:1200-10.

45. Zeng Z, Patel J, Lee S-H, McCallum M, Tyagi A, Yan M, Shea KJ. Synthetic polymer nanoparticle-polysaccharide interactions: a systematic study. J Am Chem Soc. 2012;134:2681-90.

46. Lee S-H, Hoshino Y, Randall A, Zeng Z, Baldi P, Doong R-A, Shea KJ. Engineered synthetic polymer nanoparticles as IgG affinity ligands. J Am Chem Soc. 2012;134:15765-72.

47. Wada Y, Lee H, Hoshino Y, Kotani S, Shea KJ, Miura Y. Design of multifunctional linear polymers that capture and neutralize a toxic peptide: a comparison with cross-linked nanoparticles. J Mater Chem B. 2015;3:1706-11

48. Yoshimatsu K, Koide H, Hoshino Y, Shea KJ. Preparation of abiotic polymer nanoparticles for sequestration and neutralization of a target peptide toxin. Nat Protoc. 2015;10:595-604.

49. De Sterck B, Vaneerdeweg R, Du Prez F, Waroquier M, Van Speybroeck V. Solvent effects on free radical polymerization reactions: the influence of water on the propagation rate of acrylamide and methacrylamide. Macromolecules. 2009:43:827-36.

50. Demetriades M, Leung IKH, Chowdhury R, Chan MC, McDonough MA, Yeoh KK, Tian Y-M, Claridge TDW, Ratcliffe PJ, Woon ECY, Schofield CJ. Dynamic combinatorial chemistry employing boronic acids/boronate esters leads to potent oxygenase inhibitors. Angew Chem Int Ed. 2012:51:6672-5.

51. Mondal M, Hirsch AK. Dynamic combinatorial chemistry: a tool to facilitate the identification of inhibitors for protein targets. Chem Soc Rev. 2015;44:2455-88.

52. Komáromy D, Nowak P, Otto S. Dynamic combinatorial libraries. Dynamic covalent chemistry: principles, reactions, and applications 2018; 31-119. 
53. Li J, Nowak P, Otto S. Dynamic combinatorial libraries: from explor ing molecular recognition to systems chemistry. J Am Chem Soc. 2013:135:9222-39.

54. Li J, Nowak P, Fanlo-Virgós H, Otto S. Catenanes from catenanes: quantitative assessment of cooperativity in dynamic combinatorial catenation. Chem Sci. 2014;5:4968-74.

55. Otto S, Furlan RL, Sanders JK. Selection and amplification of hosts from dynamic combinatorial libraries of macrocyclic disulfides. Science. 2002:297:590-3.

56. James LI, Beaver JE, Rice NW, Waters ML. A synthetic receptor for asymmetric dimethyl arginine. J Am Chem Soc. 2013;135:6450-5.

57. Huang R, Leung IKH. Protein-directed dynamic combinatorial chemistry: a guide to protein ligand and inhibitor discovery. Molecules. 2016;21:910.

58. Herrmann A. Dynamic combinatorial/covalent chemistry: a tool to read generate and modulate the bioactivity of compounds and compound mixtures. Chem Soc Rev. 2014;43:1899-933.

59. Ma Y, Pan G, Zhang Y, Guo X, Zhang H. Narrowly dispersed hydrophilic molecularly imprinted polymer nanoparticles for efficient molecular recognition in real aqueous samples including river water, milk, and bovine serum. Angew Chem Int Ed. 2013;52:1511-4.

60. Yang H, Yuan B, Zhang X, Scherman OA. Supramolecular chemistry at interfaces: host-guest interactions for fabricating multifunctional biointerfaces. Acc Chem Res. 2014;47:2106-15.

61. Mu B, Zhang J, McNicholas TP, Reuel NF, Kruss S, Strano MS. Recent advances in molecular recognition based on nanoengineered platforms. Acc Chem Res. 2014;47:979-88.

62. Roy N, Bruchmann B, Lehn J-M. DYNAMERS: dynamic polymers as selfhealing materials. Chem Soc Rev. 2015;44:3786-807.

63. Yang Y, Urban MW. Self-healing polymeric materials. Chem Soc Rev. 2013:42:7446-67.

64. Pan G, Guo B, Ma Y, Cui W, He F, Li B, Yang H, Shea KJ. Dynamic introduction of cell adhesive factor via reversible multicovalent phenylboronic acid/cis-diol polymeric complexes. J Am Chem Soc. 2014;136:6203-6.

65. Zeng X, Liu G, Tao W, Ma Y, Zhang X, He F, Pan J, Mei L, Pan G. A drugself-gated mesoporous antitumor nanoplatform based on ph-sensitive dynamic covalent bond. Adv Funct Mater. 2017;27:1605985.

66. Liu S, Pan J, Liu J, Ma Y, Qiu F, Mei L, Zeng X, Pan G. Dynamically PEGylated and borate-coordination-polymer-coated polydopamine nanoparticles for synergetic tumor-targeted. Chemo photothermal combination therapy. Small. 2018;14:1703968.

67. Chen H, Cheng R, Zhao X, Zhang Y, Tam A, Yan Y, Shen H, Zhang YS, Qi J, Feng Y, Liu L, Pan G, Cui W, Deng L. An injectable self-healing coordinative hydrogel with antibacterial and angiogenic properties for diabetic skin wound repair. Npg Asia Mater. 2019;11:3.

68. Wei H, Wang E. Nanomaterials with enzyme-like characteristics (nanozymes): next-generation artificial enzymes. Chem Soc Rev. 2013;42:6060-93.

69. Cormode DP, Gao L, Koo H. Emerging biomedical applications of enzyme-like catalytic nanomaterials. Trends Biotechnol. 2017;36:15-29.

70. Chang B, Zhang M, Qing G, Sun T. Dynamic biointerfaces: from recognition to function. Small. 2015;11:1097-112.

71. Bie Z, Chen Y, Ye J, Wang S, Liu Z. Boronate-affinity glycan-oriented surface imprinting: a new strategy to mimic lectins for the recognition of an intact glycoprotein and its characteristic fragments. Angew Chem Int Ed. 2015;54:10211-5.

72. Olivier GK, Cho A, Sanii B, Connolly MD, Tran H, Zuckermann RN Antibody-mimetic peptoid nanosheets for molecular recognition. ACS Nano. 2013;7:9276-86.

73. Jaegle M, Wong EL, Tauber C, Nawrotzky E, Arkona C, Rademann J. Protein-templated fragment ligations-from molecular recognition to drug discovery. Angew Chem Int Ed. 2017:56(26):7358-78.

74. Zhang M, Yan X, Huang F, Niu Z, Gibson HW. Stimuli-responsive hostguest systems based on the recognition of cryptands by organic guests. Acc Chem Res. 2014;47:1995-2005.

75. He Y, Liao S, Jia H, Cao Y, Wang Z, Wang Y. A self-healing electronic sensor based on thermal-sensitive fluids. Adv Mater. 2015;27:4622-7.

76. Montarnal D, Tournilhac F, Hidalgo M, Couturier J-L, Leibler L. Versatile one-pot synthesis of supramolecular plastics and self-healing rubbers. J Am Chem Soc. 2009;131:7966-7.
77. Guo K, Zhang DL, Zhang XM, Zhang J, Ding LS, Li BJ, Zhang S. Conductive elastomers with autonomic self-healing properties. Angew Chem. 2015;127:12295-301.

78. Harada A, Kobayashi R, Takashima Y, Hashidzume A, Yamaguchi H. Macroscopic self-assembly through molecular recognition. Nat Chem. 2011;3:34-7.

79. Miyamae K, Nakahata M, Takashima Y, Harada A. Self-healing, expansion-contraction, and shape-memory properties of a preorganized supramolecular hydrogel through host-guest interactions. Angew Chem Int Ed. 2015;54:8984-7.

80. Chi X, Yu G, Shao L, Chen J, Huang F. A dual-thermoresponsive gemini-type supra-amphiphilic macromolecular [3] pseudorotaxane based on pillar [10] arene/paraquat cooperative complexation. J Am Chem Soc. 2016;138:3168-74.

81. Yan Q, Zhao Y. ATP-triggered biomimetic deformations of bioinspired receptor-containing polymer assemblies. Chem Sci. 2015;6:4343-9.

82. Zhang W, Zhang YM, Li SH, Cui YL, Yu J, Liu Y. Tunable nanosupramolecular aggregates mediated by host-guest complexation. Angew Chem. 2016;128:11624-8.

83. Leigh DA. Genesis of the nanomachines: the 2016 Nobel prize in chemistry. Angew Chem Int Ed. 2016;55:14506-8.

84. Erbas-Cakmak S, Fielden SD, Karaca U, Leigh DA, McTernan CT, Tetlow DJ, Wilson MR. Rotary and linear molecular motors driven by pulses of a chemical fuel. Science. 2017;358:340-3.

85. Li J, Cvrtila I, Colomb-Delsuc M, Otten E, Otto S. An, "ingredients" approach to functional self-synthesizing materials: a metal-ionselective, multi-responsive, self-assembled hydrogel. Chem Eur J. 2014;20:15709-14.

86. Pappas CG, Shafi R, Sasselli IR, Siccardi H, Wang T, Narang V, Abzalimov R, Wijerathne N, Ulijn RV. Dynamic peptide libraries for the discovery of supramolecular nanomaterials. Nat Nanotechnol. 2016;11:960-7.

87. Liu Y, Stuart MC, Buhler E, Lehn JM, Hirsch AK. Proteoid dynamers with tunable properties. Adv Funct Mater. 2016;26:6297-305.

88. Pan G, Zhang Y, Guo X, Li C, Zhang H. An efficient approach to obtaining water-compatible and stimuli-responsive molecularly imprinted polymers by the facile surface-grafting of functional polymer brushes via RAFT polymerization. Biosens Bioelectron. 2010;26:976-82.

89. Wei Y, Zeng Q, Hu Q, Wang M, Tao J, Wang L. Self-cleaned electrochemical protein imprinting biosensor basing on a thermo-responsive memory hydrogel. Biosens Bioelectron. 2018;99:136-41.

90. Theato P, Sumerlin BS, O'Reilly RK, Epps TH III. Stimuli responsive materials. Chem Soc Rev. 2013;42:7055-6.

91. Chen W, Ma Y, Pan J, Meng Z, Pan G, Sellergren B. Molecularly imprinted polymers with stimuli-responsive affinity: progress and perspectives. Polymers. 2015;7:1689-715.

92. Xu S, Lu H, Zheng X, Chen L. Stimuli-responsive molecularly imprinted polymers: versatile functional materials. J Mater Chem C. 2013;1:4406-22.

93. Schild HG. Poly (N-isopropylacrylamide): experiment, theory and application. Prog Polym Sci. 1992;17:163-249.

94. Pan G, Guo Q, Cao C, Yang H, Li B. Thermo-responsive molecularly imprinted nanogels for specific recognition and controlled release of proteins. Soft Matter. 2013;9:3840-50.

95. Li S, Yang K, Deng N, Min Y, Liu L, Zhang L, Zhang Y. Thermoresponsive epitope surface-imprinted nanoparticles for specific capture and release of target protein from human plasma. ACS Appl Mater Inter. 2016:8:5747-51.

96. Li C, Ma Y, Niu H, Zhang H. Hydrophilic hollow molecularly imprinted polymer microparticles with photo-and thermoresponsive template binding and release properties in aqueous media. ACS Appl Mater Inter. 2015;7:27340-50.

97. Pan G, Guo Q, Ma Y, Yang H, Li B. Thermo-responsive hydrogel layers imprinted with RGDS peptide: a system for harvesting cell sheets. Angew Chem Int Ed. 2013;52:6907-11.

98. Culver HR, Clegg JR, Peppas NA. Analyte-responsive hydrogels: intelligent materials for biosensing and drug delivery. Acc Chem Res. 2017;50:170-8. 
99. Miyata T, Jige M, Nakaminami T, Uragami T. Tumor marker-responsive behavior of gels prepared by biomolecular imprinting. Proc Natl Acad Sci USA. 2006;103:1190-3.

100. Bai W, Gariano NA, Spivak DA. Macromolecular amplification of binding response in superaptamer hydrogels. J Am Chem Soc. 2013;135:6977-84.

101. Yoshimatsu K, Lesel BK, Yonamine Y, Beierle JM, Hoshino Y, Shea KJ. Temperature-responsive, "catch and release" of proteins by using multifunctional polymer-based nanoparticles. Angew Chem Int Ed. 2012;51:2405-8.

102. Marcos V, Stephens AJ, Jaramillo-Garcia J, Nussbaumer AL, Woltering SL, Valero A, Lemonnier J-F, Vitorica-Yrezabal IJ, Leigh DA. Allosteric initiation and regulation of catalysis with a molecular knot. Science. 2016;352:1555-9.

103. Gil-Ramírez G, Hoekman S, Kitching MO, Leigh DA, Vitorica-Yrezabal IJ, Zhang G. Tying a molecular overhand knot of single handedness and asymmetric catalysis with the corresponding pseudo-D 3-symmetric trefoil knot. J Am Chem Soc. 2016;138:13159-62.

104. Fanlo-Virgós H, Alba ANR, Hamieh S, Colomb-Delsuc M, Otto S. Transient substrate-induced catalyst formation in a dynamic molecular network. Angew Chem Int Ed. 2014;53:11346-50.

105. Zhang Q, Tiefenbacher K. Terpene cyclization catalysed inside a selfassembled cavity. Nat Chem. 2015;7:197-202.

106. Zhang Q, Catti L, Pleiss J, Tiefenbacher K. Terpene Cyclizations inside a Supramolecular Catalyst: Leaving Group-Controlled Product Selectivity and Mechanistic Studies. Soc: J. Am. Chem; 2017.

107. Alexander C, Davidson L, Hayes W. Imprinted polymers: artificial molecular recognition materials with applications in synthesis and catalysis. Tetrahedron. 2003;59:2025-57.

108. Wulff G. Enzyme-like catalysis by molecularly imprinted polymers. Chem Rev. 2002;102:1-28.

109. Guo Y, Guo T. A dual-template imprinted capsule with remarkably enhanced catalytic activity for pesticide degradation and elimination simultaneously. Chem Commun. 2013;49:1073-5.

110. Chen Z, Sellergren B, Shen X. Synergistic catalysis by "polymeric microzymes and inorganic nanozymes": the $1+1>2$ effect for intramolecular cyclization of peptides. Front Chem. 2017;5:60

111. Gu Y, Yan X, Li C, Zheng B, Li Y, Liu W, Zhang Z, Yang M. Biomimetic sensor based on molecularly imprinted polymer with nitroreductase-like activity for metronidazole detection. Biosens Bioelectron. 2016;77:393-9.

112. Liu JQ, Wulff G. Molecularly imprinted polymers with strong carboxypeptidase A-like activity: combination of an amidinium function with a zinc-ion binding site in transition-state imprinted cavities. Angew Chem Int Ed. 2004;43:1287-90.

113. Zhang H, Piacham T, Drew M, Patek M, Mosbach K, Ye L. Molecularly imprinted nanoreactors for regioselective huisgen 1, 3-dipolar cycloaddition reaction. J Am Chem Soc. 2006;128:4178-9.

114. Shen X, Huang C, Shinde S, Jagadeesan KK, Ekström S, Fritz E, Sellergren BR. Catalytic formation of disulfide bonds in peptides by molecularly imprinted microgels at oil/water interfaces. ACS Appl Mater Inter. 2016;8:30484-91.

115. Shaabani A, Afshari R, Hooshmand SE, Keramati Nejad M. Molecularly imprinted polymer as an eco-compatible nanoreactor in multicomponent reactions: a remarkable synergy for expedient access to highly substituted imidazoles. ACS Sustain Chem Eng. 2017;5:9506-16.

116. Li S, Ge Y, Tiwari A, Wang S, Turner AP, Piletsky SA. 'On/off'-switchable catalysis by a smart enzyme-like imprinted polymer. J Catal. 2011;278:173-80.

117. Zhang Z, Liu B, Liu J, Molecular imprinting for substrate selectivity and enhanced activity of enzyme mimics. Small 2017; 13, n/a-n/a.

118. Zhang Z, Zhang X, Liu B, Liu J. Molecular imprinting on inorganic nanozymes for hundred-fold enzyme specificity. J Am Chem Soc. 2017;139:5412-9.

119. Wong Y-M, Hoshino Y, Sudesh K, Miura Y, Numata K. Optimization of poly (N-isopropylacrylamide) as an artificial amidase. Biomacromol. 2014;16:411-21.

120. Boehr DD, Nussinov R, Wright PE. The role of dynamic conformational ensembles in biomolecular recognition. Nat Chem Biol. 2009;5:789-96.
121. Mahon CS, Fulton DA. Templation-induced re-equilibration in polymerscaffolded dynamic combinatorial libraries leads to enhancements in binding affinities. Chem Sci. 2013;4:3661-6.

122. Mondal M, Radeva N, Fanlo-Virgós H, Otto S, Klebe G, Hirsch AKH. Fragment linking and optimization of inhibitors of the aspartic protease endothiapepsin: fragment-based drug design facilitated by dynamic combinatorial chemistry. Angew Chem Int Ed. 2016:55:9422-6.

123. Hoshino Y, Koide H, Urakami T, Kanazawa H, Kodama T, Oku N, Shea KJ. Recognition, neutralization, and clearance of target peptides in the bloodstream of living mice by molecularly imprinted polymer nanoparticles: a plastic antibody. J Am Chem Soc. 2010;132:6644-5.

124. O'Brien J, Lee S-H, Onogi S, Shea KJ. Engineering the protein corona of a synthetic polymer nanoparticle for broad-spectrum sequestration and neutralization of venomous biomacromolecules. J Am Chem Soc. 2016;138:16604-7.

125. Li W, Dong K, Ren J, Qu X. A ß-lactamase-imprinted responsive hydrogel for the treatment of antibiotic-resistant bacteria. Angew Chem. 2016;128:8181-5.

126. Saridakis E, Khurshid S, Govada L, Phan Q, Hawkins D, Crichlow GV, Lolis E, Reddy SM, Chayen NE. Protein crystallization facilitated by molecularly imprinted polymers. Proc Natl Acad Sci USA. 2011;108:11081-6.

127. Cutivet A, Schembri C, Kovensky J, Haupt K. Molecularly imprinted microgels as enzyme inhibitors. J Am Chem Soc. 2009;131:14699-702.

128. Beierle JM, Yoshimatsu K, Chou B, Mathews MAA, Lesel BK, Shea KJ. Polymer nanoparticle hydrogels with autonomous affinity switching for the protection of proteins from thermal stress. Angew Chem Int Ed. 2014:53:9275-9.

129. Nakamoto M, Nonaka T, Shea KJ, Miura Y, Hoshino Y. Design of synthetic polymer nanoparticles that facilitate resolubilization and refolding of aggregated positively charged lysozyme. J Am Chem Soc. 2016:138:4282-5.

130. Zhang X, Parekh G, Guo B, Huang X, Dong Y, Han W, Chen X, Xiao G. Polyphenol and self-assembly: metal polyphenol nanonetwork for drug delivery and pharmaceutical applications. Future Drug Discovery, O, null.

131. Zhou M, Zhang X, Xie J, Qi R, Lu H, Leporatti S, Chen J, Hu Y. pH-sensitive poly( $\beta$-amino ester)s nanocarriers facilitate the inhibition of drug resistance in breast cancer cells. Nanomaterials. 2018:8:952.

132. Vergaro V, Scarlino F, Bellomo C, Rinaldi R, Vergara D, Maffia M, Baldassarre F, Giannelli G, Zhang X, Lvov YM, Leporatti S. Drug-loaded polyelectrolyte microcapsules for sustained targeting of cancer cells. Adv Drug Deliver Rev. 2011;63:847-64.

133. Parekh G, Shi Y, Zheng J, Zhang X, Leporatti S. Nano-carriers for targeted delivery and biomedical imaging enhancement. Therapeutic Delivery. 2018;9:451-68

134. Yin D, Wang S, He Y, Liu J, Zhou M, Ouyang J, Liu B, Chen H-Y, Liu Z. Surface-enhanced Raman scattering imaging of cancer cells and tissues via sialic acid-imprinted nanotags. Chem Commun. 2015;51:17696-9.

135. Shinde S, El-Schich Z, Malakpour A, Wan W, Dizeyi N, Mohammadi R, Rurack K, Wingren AG, Sellergren B. Sialic acid-imprinted fluorescent Core-Shell particles for selective labeling of cell surface glycans. J Am Chem Soc. 2015;137:13908-12.

136. Liu R, Cui Q, Wang C, Wang X, Yang Y, Li L. Preparation of sialic acidimprinted fluorescent conjugated nanoparticles and their application for targeted cancer cell imaging. ACS Appl Mater Inter. 2017:9:3006-15.

137. Kunath S, Panagiotopoulou M, Maximilien J, Marchyk N, Sänger J, Haupt K. Cell and tissue imaging with molecularly imprinted polymers as plastic antibody mimics. Adv Healthc Mater. 2015;4:1322-6.

138. Panagiotopoulou M, Salinas $Y$, Beyazit S, Kunath S, Duma L, Prost E, Mayes AG, Resmini M, Tse Sum Bui B, Haupt K. Molecularly imprinted polymer coated quantum dots for multiplexed cell targeting and imaging. Angew Chem. 2016;128:8384-8.

139. Guo B, Pan G, Guo Q, Zhu C, Cui W, Li B, Yang H. Saccharides and temperature dual-responsive hydrogel layers for harvesting cell sheets. Chem Commun. 2015;51:644-7.

140. Lange C, Storkebaum E, de Almodóvar CR, Dewerchin M, Carmeliet P. Vascular endothelial growth factor: a neurovascular target in neurological diseases. Neurol: Nat. Rev; 2016.

141. Ferrara N, Ten Adamis A P. Years of anti-vascular endothelial growth factor therapy. Nat Rev Drug Discov. 2016;15:385-403. 
142. Cecchini A, Raffa V, Canfarotta F, Signore G, Piletsky S, MacDonald MP, Cuschieri A. In vivo recognition of human vascular endothelial growth factor by molecularly imprinted polymers. Nano Lett. 2017;17:2307-12.

143. Koide H, Yoshimatsu K, Hoshino Y, Lee S-H, Okajima A, Ariizumi S, Narita Y, Yonamine $Y$, Weisman AC, Nishimura Y. A polymer nanoparticle with engineered affinity for a vascular endothelial growth factor (VEGF165). Nat Chem. 2017:9:715.

144. Zhang Y, Deng C, Liu S, Wu J, Chen Z, Li C, Lu W. Active targeting of tumors through conformational epitope imprinting. Angew Chem Int Ed. 2015;54:5157-60.

145. Peng S, Wang Y, Li N, Li C. Enhanced cellular uptake and tumor penetration of nanoparticles by imprinting the "hidden" part of membrane receptors for targeted drug delivery. Chem Commun. 2017;53:11114-7.

146. Yin D, Li X, Ma Y, Liu Z. Targeted Cancer Imaging and Photothermal Therapy via Monosaccharide-Imprinted Gold Nanorods. Commun: Chem; 2017.

147. Mager MD, LaPointe V, Stevens MM. Exploring and exploiting chemistry at the cell surface. Nat Chem. 2011;3:582.

148. Liu L, Tian X, Ma Y, Duan Y, Zhao X, Pan G. A versatile dynamic musselinspired biointerface: from specific cell behavior modulation to selective cell isolation. Angew Chem Int Ed. 2018;57:7878-82.
149. Robertus J, Browne WR, Feringa BL. Dynamic control over cell adhesive properties using molecular-based surface engineering strategies. Chem Soc Rev. 2010;39:354-78.

150. Ma Y, Tian X, Liu L, Pan J, Pan G. Dynamic synthetic biointerfaces: from reversible chemical interactions to tunable biological effects. Acc Chem. Res. 2019.

151. Pan G, Sun S, Zhang W, Zhao R, Cui W, He F, Huang L, Lee S-H, Shea KJ, Shi Q. Biomimetic design of mussel-derived bioactive peptides for dual-functionalization of titanium-based biomaterials. J Am Chem Soc. 2016;138:15078-86.

152. Nishino H, Huang CS, Shea KJ. Selective protein capture by epitope imprinting. Angew Chem Int Ed. 2006;45:2392-6.

153. Pan G, Shinde S, Yeung SY, Jakštaitè M, Li Q, Wingren AG, Sellergren B. An epitope-imprinted biointerface with dynamic bioactivity for modulating cell-biomaterial interactions. Angew Chem Int Ed. 2017:56:15959-63.

\section{Publisher's Note}

Springer Nature remains neutral with regard to jurisdictional claims in published maps and institutional affiliations.
Ready to submit your research? Choose BMC and benefit from:

- fast, convenient online submission

- thorough peer review by experienced researchers in your field

- rapid publication on acceptance

- support for research data, including large and complex data types

- gold Open Access which fosters wider collaboration and increased citations

- maximum visibility for your research: over $100 \mathrm{M}$ website views per year

At $\mathrm{BMC}$, research is always in progress.

Learn more biomedcentral.com/submissions 